\title{
On latency of multiple zonal jets in the oceans
}

\author{
P. Berloff ${ }^{1} \dagger$, S. Karabasov ${ }^{2}$, J. T. Farrar ${ }^{3}$ and I. Kamenkovich ${ }^{4}$ \\ ${ }^{1}$ Department of Mathematics, Grantham Institute for Climate Change, Imperial College London, \\ London SW7 2AZ, UK \\ 2 Department of Engineering, Whittle Laboratory, University of Cambridge, Cambridge CB3 ODY, UK \\ ${ }^{3}$ Physical Oceanography Department, Woods Hole Oceanographic Institution, MA 02543, USA \\ ${ }^{4}$ RSMAS, University of Miami, FL 33149, USA
}

(Received 24 November 2010; revised 6 August 2011; accepted 8 August 2011; first published online 27 September 2011)

\begin{abstract}
Most of the nearly zonal, multiple, alternating jets observed in the oceans are latent, that is, their amplitudes are weak relative to the ambient mesoscale eddies. Yet, relatively strong jets are often observed in dynamical simulations. To explore mechanisms controlling the degree of latency, we analyse solutions of an idealized, eddy-resolving and flat-bottom quasigeostrophic model, in which dynamically generated mesoscale eddies maintain and interact with a set of multiple zonal jets. We find that the degree of the latency is controlled primarily by the bottom friction: the larger the friction parameter, the more latent are the jets; and the degree of the latency is substantial for a realistic range of the oceanic bottom friction coefficient. This result not only provides a plausible explanation for the latency of the oceanic jets, but it may also be relevant to the prominent atmospheric multiple jets observed on giant gas planets, such as Jupiter. We hypothesize that these jets can be so strong because of the relative absence of the bottom friction. The mechanism controlling the latency in our solutions is understood in terms of the changes induced in the linear eigenmodes of the time-mean flow by varying the bottom friction coefficient; these changes, in turn, affect and modify the jets. Effects of large Reynolds numbers on the eddies, jets, and the latency are also discussed.
\end{abstract}

Key words: geostrophic turbulence, quasi-geostrophic flows, waves in rotating fluids

\section{Introduction}

\subsection{Background}

Over the last few years, observations of multiple, alternating, nearly zonal features in time-averaged properties of the global ocean have come from sea-surface altimetry measurements, float trajectories, and chemical tracer distributions (Hogg \& Owens 1999; Maximenko, Bang \& Sasaki 2005; Maximenko et al. 2008; Ollitrault et al. 2006; Sokolov \& Rintoul 2007a,b, 2009; Huang et al. 2007; Herbei, McKeague \& Speer 2008; Ivanov, Collins \& Margolina 2008; van Sebille, Kamenkovich \& Willis 2011), as well as from eddying solutions of primitive-equation general circulation models (GCMs) (Sinha \& Richards 1999; Nakano \& Hasumi 2005; Richards et al. 2006; Huang et al. 2007; Kamenkovich, Berloff \& Pedlosky 2009a,b; Levy et al. 2010; Melnichenko et al. 2010). These features can be viewed as time-mean zonally elongated anomalies on large-scale background flows such as, for example, midlatitude 
gyres and the Antarctic Circumpolar Current, and they have been reported to exist in all oceans, except for the Arctic. Use of the term 'jets' to describe these latent oceanic flows is common but somewhat imprecise, because these flows are poorly visible in the instantaneous flow.

Typical multi-year time-average zonal velocities of these oceanic jets are a few centimetres per second (the time-averaging interval of the observations is limited to less than two decades of the satellite altimetry record, and typical time spans of the comprehensive eddy-resolving GCM solutions are limited to a few decades). Multiple zonal jets are not visible in the instantaneous oceanic flow field, which is instead dominated by long-lived, westward-propagating eddies (e.g. Chelton, Schlax \& Samelson 2011). Thus, the observed jets are 'latent', in the sense that they are weaker than the ambient mesoscale eddies, and they become apparent only after long time averaging.

Away from the equatorial zone, the oceanic jet features are so weak relative to the velocities of mesoscale eddies that Schlax \& Chelton (2008) argued that the appearance of zonal jets in a time average of oceanic data over a limited time period (e.g. a decade) is likely an artifact of inadequate averaging over the more energetic, westward-moving eddies. Whether the oceanic zonal-jet features are real dynamical features or simply a result of averaging over energetic, westward-moving eddies is beside the point of this paper: it suffices to say that the oceanic jets are highly latent. However, numerical simulations with nonlinear dynamical models having parameter values nominally appropriate for the ocean readily produce highly non-latent zonal jets (i.e. 'manifest' jets) that are visible in the instantaneous flow field (e.g. Panetta 1993; Galperin et al. 2004; Berloff, Kamenkovich \& Pedlosky 2009b; Liu \& Schneider 2011), ones qualitatively more similar to the jets observed on Jupiter (e.g. Kondratyev \& Hunt 1982).

This situation raises a fundamental question about these jets: Why are multiple zonal jets obviously present in some flow regimes and not obviously present in others? The purpose of this study is to understand what dynamical processes can cause these jets to be more or less apparent in the presence of the eddy field. With an idealized, nonlinear numerical model, we will show that, depending on basic parameters of the flow (Reynolds number and bottom-friction coefficient), the flow can exhibit different degrees of jet latency, from flows with strong jets that are apparent in the instantaneous flow field to flows with a vigorous eddy field and no jets at all. This variation in the degree of latency is the focus of this paper. In the paper we refer to the weakness or strength of the time-mean jets relative to the transient mesoscale eddies as the 'latency', we provide quantitative measure of it, and focus on the physics underpinning the latency in several flow regimes.

There are comprehensive reviews of such jets and associated theoretical ideas, deeply rooted in the theory of geostrophic and anisotropic turbulence (e.g. Rhines 1994; Dritschel \& McIntyre 2008). Since the ground-breaking work of Rhines (1975), theoretical attention has been paid mostly to barotropic (i.e. purely two-dimensional) models, driven either by decaying turbulence or by spatially homogeneous, smallscale random forcing meant to represent baroclinic eddy effects, as first done by Williams (1978). In these models the jets can be generated by an anisotropic inverse energy cascade (Vallis \& Maltrud 1993; Chekhlov et al. 1996; Danilov \& Gurarie 2004; Danilov \& Gryanik 2004; Galperin et al. 2004; Galperin, Sukoriansky \& Dikovskaya 2010), characterized by non-local spectral nonlinear interactions (Balk, Nazarenko \& Zakharov 1990). Similar equivalent-barotropic models have been also considered (Smith 2004; Theiss 2004; Dritschel \& Scott 2011). Some studies with 
more dynamically consistent forcings have focused on the jets emerging in a zonal channel from baroclinically unstable zonal flow (Panetta 1993; Treguier \& Panetta 1994; Kaspi \& Flierl 2007; Thompson \& Young 2007; Berloff, Kamenkovich \& Pedlosky 2009a; Berloff et al. 2009b), and Hristova, Pedlosky \& Spall (2008) suggested that multiple zonal jets can be generated by instability of the meridional flow.

In the barotropic models, the only eddy effect maintaining the jets is associated with divergence of the eddy momentum fluxes, or, equivalently, the eddy relative vorticity fluxes. The meridional scale of the barotropic jets is thought to be the physical scale at which the inverse energy cascade in turbulence is partially 'arrested' by propagating Rossby waves (e.g. Rhines 1994). This so-called Rhines scale is determined by a balance between the nonlinear advection and the linear meridional advection of the planetary vorticity. Alternative scalings corresponding to the transition from the turbulence to Rossby waves have been also proposed (Vallis \& Maltrud 1993; Sukoriansky, Dikovskaya \& Galperin 2007). Also, it is argued that although eddies at the Rhines scale possess most of the eddy energy, the jets are dynamically maintained by smaller eddies (Huang \& Robinson 1998).

In addition to the Rhines scale, more realistic baroclinic models (i.e. vertically stratified ones containing both barotropic and baroclinic dynamics) have other fundamental horizontal length scales associated with the vertical stratification (i.e. baroclinic deformation radii), and there are important dynamical interactions between the vertical barotropic and baroclinic modes. It has been suggested that, in the presence of bottom friction, baroclinic jets are associated with baroclinic-barotropic interactions that break down the inverse cascade arguments (Thompson \& Young 2007). The baroclinic models further suggest that even the barotropic components of the zonal jets are maintained by the systematic rectified action of the baroclinic eddies, through their time-mean nonlinear interactions (Berloff et al. 2009a; Kamenkovich et al. 2009a). Finally, the baroclinic models suggest that not only are baroclinic-barotropic interactions at the heart of the eddy-jet interactions, but also that the baroclinic component of the jets can be maintained either by eddy momentum fluxes or by eddy buoyancy fluxes, depending on the direction of the unstable background flow that feeds the eddies. The analysis of the corresponding eddies suggests that instead of an inverse energy cascade from the small scales of the external forcing, as happens in the barotropic models, the baroclinic-model dynamics is dominated by nonlinear interactions of the linear eigenmodes that appear at mesoscales (Berloff et al. 2009a,b). Thus, the baroclinic dynamics has some fundamental differences from the barotropic one.

Several studies provide evidence for highly anisotropic and spatially inhomogeneous mixing in the presence of the jets. The 'staircase' idea suggests that some parts of the jets act as narrow barriers to the material transport induced by the flow, and these barriers separate relatively broad zonal bands characterized by intense eddy-induced mixing of material properties and partial homogenization of potential vorticity (PV) (McIntyre 1982; Baldwin et al. 2007). Supporting evidence for this comes from some numerical models (e.g. Greenslade and Haynes 2008; Dritschel \& McIntyre 2008), observations (Marshall et al. 2006), and laboratory experiments (Read et al. 2007), and this evidence is consistent with the observed atmospheric jets on the giant gas planets. However, many other studies do not find a sharp staircase (Panetta 1993; Thompson \& Young 2007; Berloff et al. 2009a; Kamenkovich et al. 2009a); instead, they find more gradual meridional variations in meridional transport and other flow properties, more consistent with oceanic observations. Oceanic observations of zonally banded features 
in chlorophyll concentration (Sokolov \& Rintoul 2007b) and sea-surface temperature (Buckingham, Cornillon \& Obenour 2011) suggest the existence of weak mixing zones separated by weak barriers embedded in the jets. Finally, it is argued that anisotropic transport and mixing associated with the oceanic jets (Kamenkovich et al. $2009 b$ ) can be important for distributions of chemical tracers influencing the global climate, and therefore developing various diagnostics for this transport and mixing is an active research area (e.g. Hughes, Thompson \& Wilson 2010; Beron-Vera et al. 2010). Overall, the issue of the contrast between the partial barriers and mixing zones, as well as the corresponding distinctions between the oceanic and atmospheric jets, will be resolved only with progress toward more physically complete models and with more complete understanding of the various physical processes that affect the jets. A goal of this study is investigation of the jets and eddies in a model which is idealized, but with very nonlinear and detailed eddy dynamics.

In summary, past studies have not yielded a universally accepted view on the origins and dynamics of multiple zonal jets observed in the oceans and in the atmospheres of giant gas planets. However, nearly all theories argue that the jets are a nonlinear phenomenon driven by mesoscale eddies in the presence of the background gradient of the planetary vorticity. The lack of clear physical understanding of the dynamics shaping the jets and eddies calls for thorough investigation in progressively more realistic models.

The essential and novel part of this paper is that it focuses on the jet latency - an important property of multiple jets that compares their strength to that of the ambient mesoscale eddies - in the context of a geometrically idealized model that has very detailed eddy dynamics. Qualitatively, the more latent the jets are, the more they are obscured by the eddy field, and the less visible they are in the flow. A simple quantitative measure of the latency is proposed. Solutions of a two-layer, quasigeostrophic model are explored for a broad range of dissipative parameters, and the associated complex effects of dissipation on the jets and eddies are discussed in detail. We argue that the mechanisms underpinning the latency, as well as some other properties of the solutions, can be understood in terms of the properties of the linear eigenmodes of the time-mean flow. These properties involve dispersion relationships and spatial patterns of the eigenmodes, as well as their nonlinear self-interactions and the efficiency of these interactions in maintaining the jets. In this theoretical framework, we build our work on the results of Berloff et al. (2009b), who focused on the formation and meridional scale of the jets. The novelty here is in the focus on the effects of dissipation and large Reynolds number on the latency. We interpret these effects in terms of the linear eigenmodes and their interactions. To our knowledge, the importance of the underlying linear eigenmodes in shaping some of the key properties of the multiple jets and ambient eddies has been emphasized only in Berloff $e t$ al. (2009b) and Yoo \& Lee (2010). Our present advance is facilitated by a new and very efficient advection scheme (Karabasov, Berloff \& Goloviznin 2009). A particular advantage of this scheme is the ability to carry out accurate simulations of baroclinic jets and ambient eddies at relatively large Reynolds numbers, and thus on a new level of dynamical realism. From analysis of the model solutions, we argue that the main factor controlling the latency is the magnitude of the bottom friction, whereas the degree of the solution nonlinearity (i.e. the Reynolds number) is a secondary controlling factor. 


\subsection{Dynamical model of the jets}

The model of the multiple jets used in this study is similar to the model of Panetta (1993) that was used for simulating the atmospheric jets. Our model uses different numerical algorithms and much finer horizontal grid resolution, which allow us to reach unprecedentedly large Reynolds numbers (for this kind of model), allowing a high degree of nonlinearity in the dynamics. The model incorporates an idealized zonal background flow, which is the ultimate source of the flow instabilities and eddies. Such background flow can crudely represent either a large-scale part of the Antarctic Circumpolar Current or nearly zonal parts of the wind-driven midlatitude gyres, away from the meridional boundaries. In all of these large-scale currents, recent observational studies have found robust but latent multiple jets. Our model parameters and set-up are more oceanic than atmospheric; however, we checked that, with the atmospheric parameters of Panetta (1993), the 'atmospheric' flow solutions are qualitatively similar. Therefore, our main conclusions are relevant not only to the oceanic but also to the atmospheric jets.

The horizontally uniform background flow in the model is zonal (i.e. eastward or westward), and it has a vertical shear that makes it baroclinically unstable to small perturbations. In the statistically equilibrated solutions, the background flow is a dynamically consistent source of energy for the multiple jets and the ambient mesoscale eddies. The model is configured in a zonally periodic channel with vertical sidewalls and flat bottom, but, in order to estimate dependence of the results on the presence of sidewalls, we also explored a doubly periodic configuration of the model. Both the uniformity of the background flow and the absence of the bottom topography allowed us to avoid introducing additional length scales in the problem. The motivation for this idealization is to establish a simple, but physically relevant, starting point, so that more physical complexity can be systematically added later on.

The model domain has a meridional width of $L_{y}=3600 \mathrm{~km}$, which typically allows development of up to 10 pairs of jets, and a zonal period of $L_{x}=2 L_{y}$ (by a 'pair of jets', we mean one flowing east relative to the background flow, and one flowing west relative to the background flow). The mid-channel Coriolis parameter is $f_{0}=0.83 \times 10^{-4} \mathrm{~s}^{-1}$ (corresponding to $45^{\circ} \mathrm{N}$ ), the background planetary vorticity gradient is $\beta=2 \times 10^{-11} \mathrm{~m}^{-1} \mathrm{~s}^{-1}$, and the governing equations are formulated on the $\beta$-plane. The vertical stratification is simple but permits the essential baroclinic instability mechanism: there are two stacked isopycnal (i.e. constant-density) layers with equilibrium thicknesses $H_{1}=1 \mathrm{~km}$ (upper layer) and $H_{2}=3 \mathrm{~km}$ (deep layer). The corresponding stratification parameters in the governing equations are

$$
S_{1}=\frac{f_{0}^{2}}{H_{1} g_{1}^{\prime}}, \quad S_{2}=\frac{f_{0}^{2}}{H_{2} g_{1}^{\prime}},
$$

where $g_{1}^{\prime}$ is the reduced-gravity coefficient associated with the density jump between the isopycnal layers. We chose $g_{1}^{\prime}$ so that the first baroclinic Rossby deformation radius, $R d_{1}=\sqrt{g_{1}^{\prime} H_{1} H_{2}} /\left(f_{0} \sqrt{H_{1}+H_{2}}\right)$, which is the fundamental length scale for the jets and ambient eddies (Berloff et al. 2009b), is $25 \mathrm{~km}$. A practical advantage of the two-layer formulation of the model is that it eliminates the second and higher baroclinic modes, which not only complicate the dynamics but also require substantially finer spatial resolution of the model, because of their smaller deformation radii (Barnier, Hua \& Le Provost 1991).

The bottom friction coefficient, $\gamma$, is one of the key parameters of this study. Its importance in controlling large-scale circulation is recognized (e.g. Dewar 1998), 
but its effect on the latency of multiple zonal jets has never been studied. In the model, the bottom friction acts as linear drag on the bottom layer velocity, and, in terms of the vorticity dynamics, this is equivalent to damping of the deep-layer relative vorticity. Effects of nonlinear bottom friction are not considered here, but they are expected to be moderate (Arbic \& Scott 2008).

The governing quasigeostrophic PV equations (e.g. Pedlosky 1987) for two dynamically active isopycnal layers are

$$
\begin{gathered}
\frac{\partial q_{1}}{\partial t}+J\left(\psi_{1}, q_{1}\right)+\beta \frac{\partial \psi_{1}}{\partial x}=v \nabla^{4} \psi_{1}, \\
\frac{\partial q_{2}}{\partial t}+J\left(\psi_{2}, q_{2}\right)+\beta \frac{\partial \psi_{2}}{\partial x}=v \nabla^{4} \psi_{2}-\gamma \nabla^{2} \psi_{2},
\end{gathered}
$$

where the layer index starts from the top; the $x$ and $y$ coordinates correspond to the zonal and meridional directions, respectively; $J($,$) is the Jacobian operator; and$ the terms with $v$ and $\gamma$ are the (Newtonian) lateral eddy viscosity and the bottom friction, respectively. Isopycnal PV anomalies, $q_{i}$, are related to isopycnal velocity streamfunctions, $\psi_{i}$, through the elliptic sub-problem for PV inversion:

$$
\begin{aligned}
& q_{1}=\nabla^{2} \psi_{1}+S_{1}\left(\psi_{2}-\psi_{1}\right), \\
& q_{2}=\nabla^{2} \psi_{2}+S_{2}\left(\psi_{1}-\psi_{2}\right) .
\end{aligned}
$$

The isopycnal velocity components are found from the corresponding velocity streamfunctions:

$$
u_{i}=-\frac{\partial \psi_{i}}{\partial y}, \quad v_{i}=\frac{\partial \psi_{i}}{\partial x} .
$$

No-slip lateral-boundary conditions are used on the sidewalls, and the mass and momentum constraints are imposed following McWilliams (1977).

The forcing in the governing equations is introduced through an imposed, vertically sheared, baroclinically unstable background flow (e.g. Haidvogel \& Held 1980; Panetta 1993):

$$
\Psi_{i}=-U_{i} y ; \quad \psi_{i} \longrightarrow \Psi_{i}+\psi_{i},
$$

where $U_{i}$ are the background zonal-velocity parameters of the problem. We consider only two dynamically different regimes (Berloff et al. 2009a,b): eastward-background (EB) flow with $U_{1}=6 \mathrm{~cm} \mathrm{~s}^{-1}$ and westward-background (WB) flow with $U_{1}=$ $-3 \mathrm{~cm} \mathrm{~s}^{-1}$, respectively, with $U_{2}=0$ for both cases. Since the EB flow tends to be more stable (Pedlosky 1987), it requires a larger vertical shear to meet the necessary condition for instability. The only forcing of the system is the internally generated eddy forcing associated with transfer of energy from the imposed background flow to the instabilities and eddies, and from them to other motions, including zonal jets.

Given (1.7), the governing equations are re-written with respect to the fluctuations, $\psi_{i}$ and $q_{i}$, around the background flow, then discretized and solved numerically. Although the model shell is borrowed from Berloff et al. (2009a), its numerical core is completely overhauled to achieve much higher accuracy and convergence of solutions for the same computational cost. In particular, the new scheme allows simulation of flow regimes at unprecedentedly large Reynolds number,

$$
R e=\frac{U_{1} R d_{1}}{v},
$$


achieved by lowering eddy viscosity, $v$, to values deemed more suitable for representing effects of unresolved small-scale motions. (Eddy viscosity in our model parametrizes the momentum transfer by such unresolved small-scale motions, and the corresponding viscosity coefficient in the oceans is estimated to be of the order of 1-10 $\mathrm{m}^{2} \mathrm{~s}^{-1}$ (Muller 1976).) The typical price to pay for achieving larger Reynolds numbers is spatial refinement of the numerical grid, required for an accurate and numerically converged (i.e. approximating the governing equations) solution, and the resulting computational overhead. For typical eddy viscosity and diffusivity values used in ocean modelling, the grid spacing requirements are very demanding (e.g. Wallcraft et al. 2005). To partially overcome these limitations, we used a highresolution numerical scheme (discussed below) that permits use of a substantially coarser grid for the same degree of numerical accuracy and convergence. The previous studies of Berloff et al. $(2009 a, b)$ used $v=100 \mathrm{~m}^{2} \mathrm{~s}^{-1}$ and the same $R d_{1}$ and $U_{1}$, giving $R e=15$ (for the EB flow, whereas for the WB flow $R e$ is half as large). In the present study, which also considers four times larger domain for a more realistic population of jets, we reduced $v$ to $2 \mathrm{~m}^{2} \mathrm{~s}^{-1}$, giving $R e=750$ (for the EB flow, whereas for the WB flow it is $R e=375$ ). In our moderate-Re solutions $v=50 \mathrm{~m}^{2} \mathrm{~s}^{-1}$, giving $R e=30$ (for the EB flow, whereas for the WB flow it is $R e=15$ ).

The governing equations (1.2)-(1.5) with substitution (1.7) are discretized with a high-resolution, second-order finite difference method, following Karabasov et al. (2009). The key aspect of the new numerical core is its advanced advection scheme called CABARET, which stands for compact accurately boundary adjusting highresolution technique, and its mathematical underpinning is in Karabasov \& Goloviznin (2009). CABARET is low-dispersive, non-dissipative, and enstrophy-conserving in the linear-advection limit; its computational stencil is very local, with only one cell in space and time; and for highly nonlinear flows it employs flux correction based on the maximum principle. The CABARET compactness and ability to accurately handle strong gradients without producing spurious oscillations make it not only very different from the standard pseudo-spectral methods but also computationally cost-effective for turbulent flows. The CABARET flux correction implies that all unresolved anomalies that cannot be transported on the given grid, while keeping their concentrations positive, are removed without affecting the resolved scales. Since this removal is accomplished implicitly, CABARET falls in the monotonically integrated large-eddy simulation (MILES) framework (e.g. review by Fureby \& Grinstein 2002). In the MILES approach, the capability of capturing sharp velocity gradients for the smallest resolved scales is very important for representing these scales, and the low-dispersive and low-dissipative properties of CABARET allow extension of this capability down to the Nyquist frequency (Goloviznin et al. 2011).

Within the CABARET method, the prognostic equations are marched in time with the predictor-corrector scheme and the adjustable time step based on the Courant condition. The elliptic sub-problem for PV inversion (1.4)-(1.5) is diagonalized and solved for the corresponding velocity streamfunctions on each time step, by a direct Swarztrauber (1977) solver. The horizontal grid, with $2048 \times 1025$ points, has uniform resolution of about $3.7 \mathrm{~km}$, and numerical convergence of the solutions has been tested by several simulations with $4096 \times 2049$ grid spacing. The latter simulations are qualitatively similar, although they have small quantitative differences of about $10 \%$ in some key diagnostics. Although the $3.7 \mathrm{~km}$ resolution is much better than typical resolutions of the modern 'eddy-resolving' ocean models, it still amounts to less than 7 grid points over $R d_{1}$. This resolution would not result in accurate representation of advection and a numerically converged solution if traditional advection schemes 
were used, but it does with CABARET. So, for each set of parameters the model is integrated in time until a statistically equilibrated solution regime is reached (typically after 20-40 years of integration), and the next 60 years are saved for analysis.

\section{Nonlinear solutions}

In this section we discuss solutions of the nonlinear dynamical model with different values of the bottom friction coefficient, and in the large- and small-Re flow regimes corresponding to $v=2$ and $50 \mathrm{~m}^{2} \mathrm{~s}^{-1}$, respectively. Our goal here is to establish phenomenology and quantify the latency properties of the solutions.

The solutions described below clearly exhibit different degrees of latency, with some parameter regimes giving jets that are clearly visible and others giving a vigorous vortex-like eddy field and no jets, so we need a quantitative measure of latency. Similar to Kamenkovich et al. (2009a), we introduce a simple quantitative measure, $\Lambda_{i}$, the latency coefficient, defined as square root of the ratio of the two area-averaged variances:

$$
\Lambda_{i}=\left\langle\frac{\Sigma_{i}^{\prime}}{\Sigma_{i}}\right\rangle^{1 / 2}, \quad \Sigma_{i}^{\prime}=\frac{1}{L_{x} L_{y}} \iint q_{i}^{\prime 2} \mathrm{~d} x \mathrm{~d} y, \quad \Sigma_{i}=\frac{1}{L_{y}} \int \bar{q}_{i}^{2} \mathrm{~d} y,
$$

where variance of the PV anomaly associated with zonally averaged jets (jet variance) is in the denominator (bar denotes zonal average), variance of the remainder of the PV anomaly (eddy variance) is in the numerator, and angular brackets denote (long) time averaging. Large $\Lambda_{i}$ corresponds to the 'latent' jets that are weak relative to the eddies. The latency coefficient is defined for each isopycnal layer, and for the two-layer flow it is conveniently represented by a pair of numbers, $\left[\Lambda_{1}, \Lambda_{2}\right]$. We calculated the latency coefficient not only for all reference solutions discussed below but also for several intermediate values of $\gamma$, in order to establish empirical dependence of $\Lambda$ on the bottom friction (figure 6).

We focus on several reference solutions, corresponding to small, large, and intermediate values of $\gamma$, for each case of the background flow and $R e$. The upperocean PV anomalies of the EB and WB large- $R e$ solutions are shown in figures 1 and 2, respectively, and examples of the deep-ocean PV distributions are shown in figure 3. The effect of large $R e$ on the solutions is discussed later in this section, and a theoretical explanation of the growth of latency with bottom friction is given in $\S 4$. The solutions are illustrated with their PV anomalies, which are related to the velocity streamfunctions through the PV inversion relations (1.4)-(1.5). The velocity streamfunctions (figure 4) are typically much smoother than the corresponding PV fields. The PV anomalies, $q_{i}$, are defined with respect to the background PV distributions, which are zonally uniform and meridionally linear (figure 5):

$$
Q_{1}(y)=\left(\beta+S_{1} U_{1}\right) y, \quad Q_{2}(y)=\left(\beta-S_{2} U_{1}\right) y .
$$

The corresponding PV gradients change sign in the vertical, as required for background flows that are baroclinically unstable (Pedlosky 1989). Below, we discuss properties of the reference solutions in detail.

The EB small-, medium-, and large- $\gamma$ cases correspond to the bottom friction coefficient $\gamma=10^{-8}, 10^{-7}, 10^{-6} \mathrm{~s}^{-1}$, respectively, and the oceanographically most relevant regime is in the middle of this range of values. The small- $\gamma$ solution is characterized by multiple jets that are strong relative to the background flow, with a marked asymmetry between eastward and westward jets. The cores of the eastward jets act as partial, meandering transport barriers separating opposite-sign PV anomalies, 
(a)

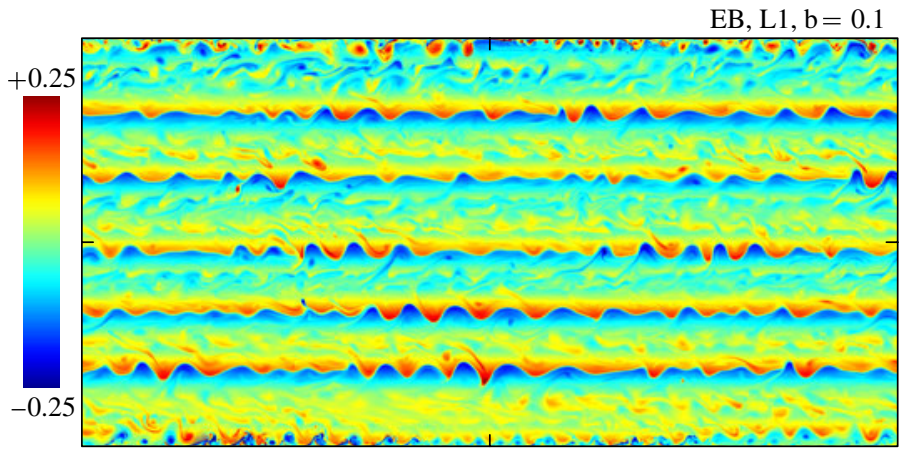

(b)
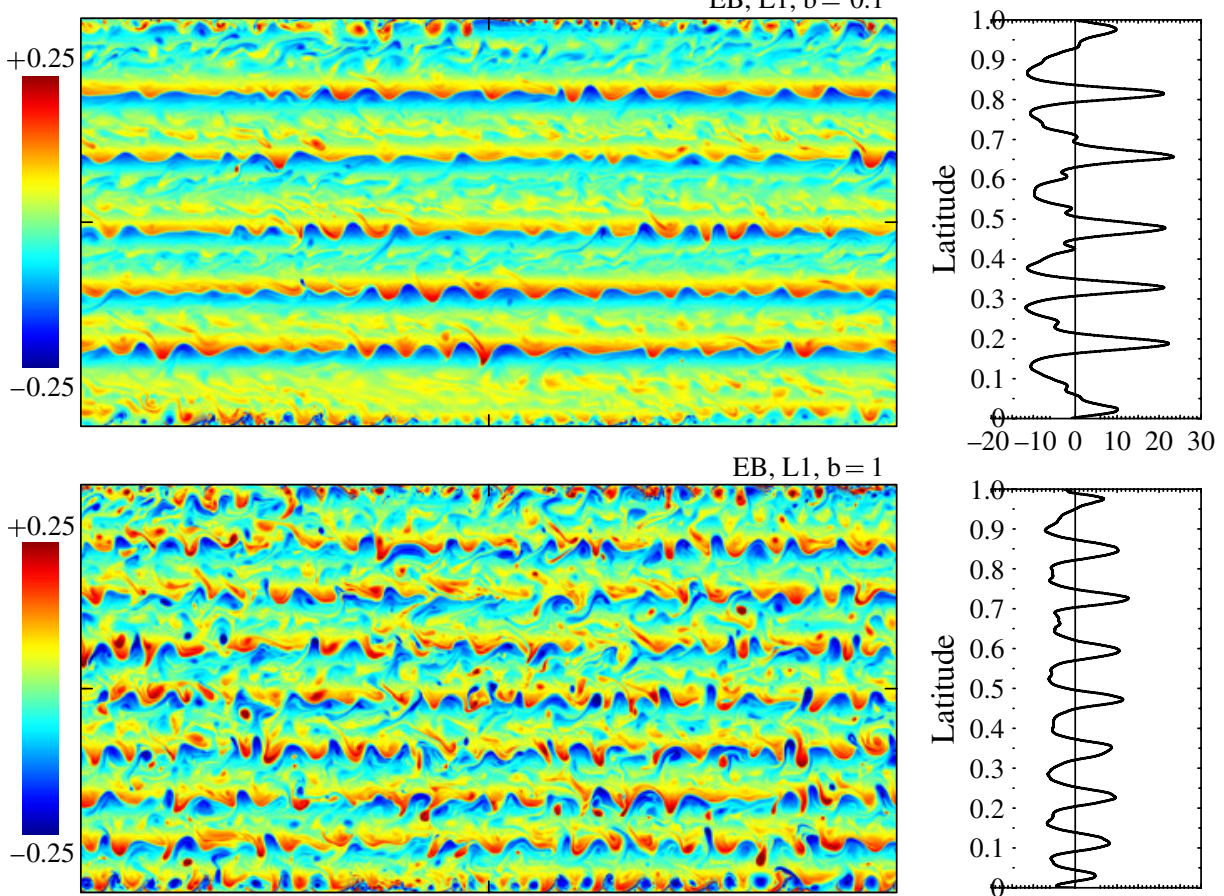

(c)
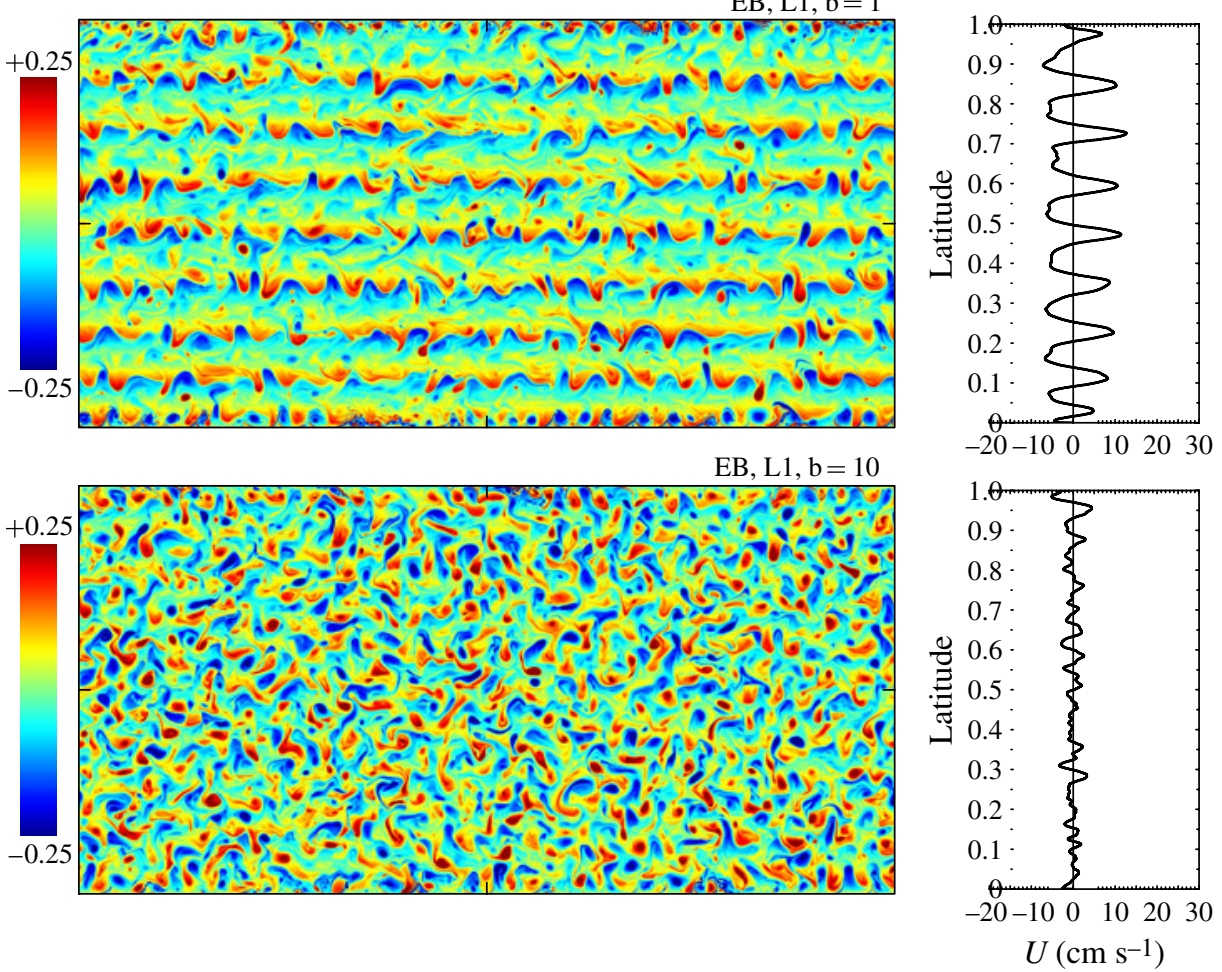

FIGURE 1. Dependence of the multiple jets and eddies on bottom friction: EB flow case, large $R e$. Upper-ocean PV anomalies (left panels, in colour) and the corresponding zonally averaged zonal velocity profiles (right panels) are shown for three different values of bottom friction, $\gamma$ : (a) $10^{-8},(b) 10^{-7}$, and (c) $10^{-6} \mathrm{~s}^{-1}$. Potential vorticity anomaly values are normalized by the Coriolis parameter. Each run has $R e=750$ (eddy viscosity is $v=2 \mathrm{~m}^{2} \mathrm{~s}^{-1}$ ).

whereas westward jets correspond to relatively mixed PV zones. This solution is still substantially different from the ideal 'PV staircase' limit proposed by Dritschel \& McIntyre (2008) for the $R e \rightarrow \infty$ situation: the PV profile of the solution in the upper layer (figure 5) and, even more so, in the deep layer (not shown) is similar to the 'PV washboard' from Berloff et al. (2009a). However, at larger Re we see a clear tendency towards the 'PV staircase', because the PV contrast across the eastward jet cores becomes more pronounced. (This is also evident from calculating semi-Lagrangian 
(a)
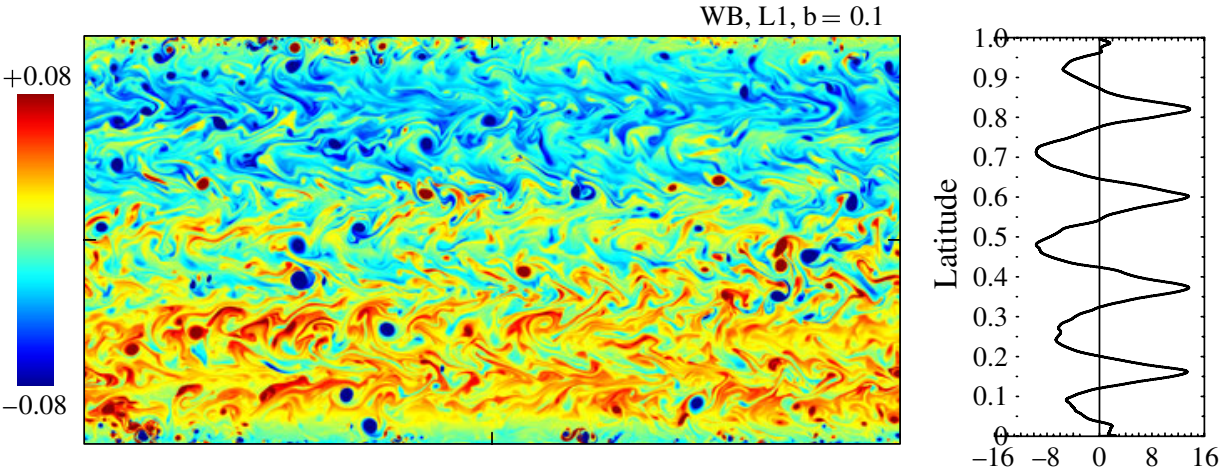

(b)
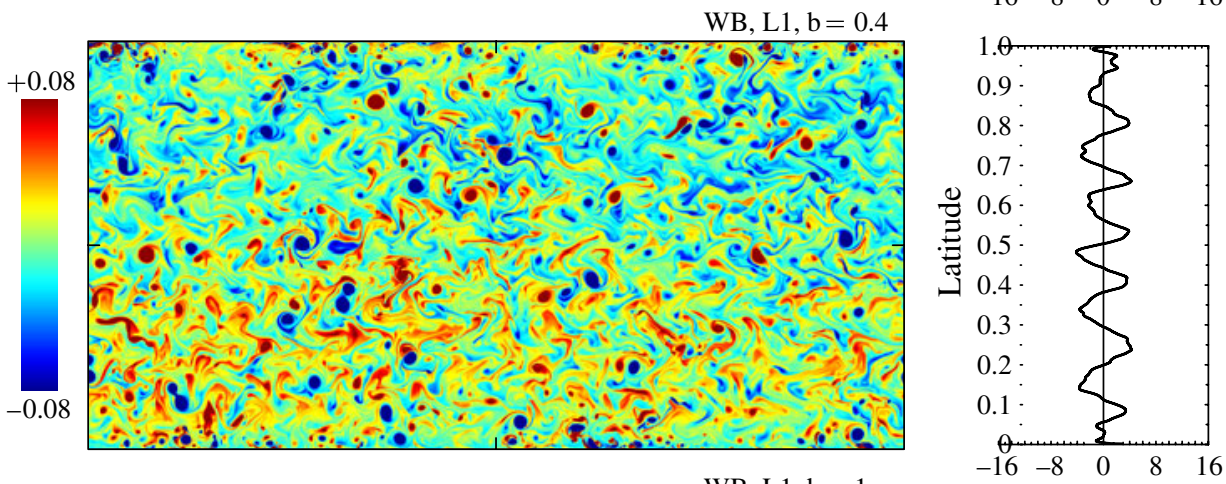

(c)
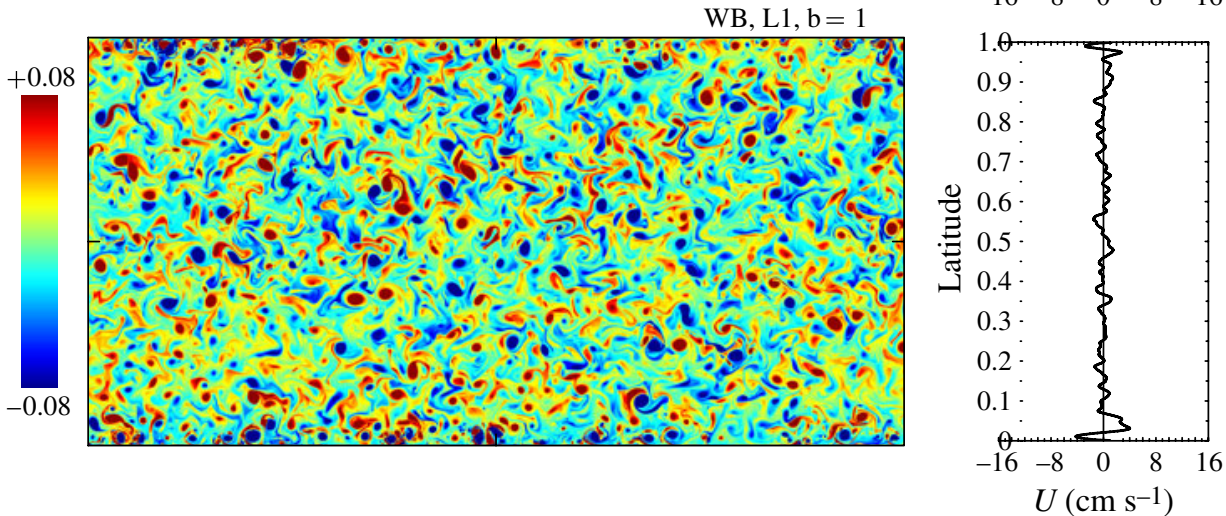

FIGURE 2. Dependence of the multiple jets and eddies on bottom friction: WB flow case, large $R e$. Upper-ocean PV anomalies (left panels, in colour) and the corresponding zonally averaged zonal velocity profiles (right panels) are shown for 3 different values of bottom friction, $\gamma$ : (a) $10^{-8},(b) 4 \times 10^{-8}$, and $(c) 10^{-7} \mathrm{~s}^{-1}$. Potential vorticity anomaly values are normalized by the Coriolis parameter. Each run has $R e=375$ (eddy viscosity is $\left.v=2 \mathrm{~m}^{2} \mathrm{~s}^{-1}\right)$.

meridional PV profiles, following Dritschel \& McIntyre (2008).) With medium $\gamma$, the amplitude of the jets is significantly reduced, the eastward jet cores are relatively convoluted and incoherent, and the eddies are stronger and frequently travel across the jet cores. Overall, the jets become significantly more latent. The large- $\gamma$ regime is characterized by an almost complete destruction of the basin-wide jets, as seen both in the time-mean fields and snapshots, and the resulting flow pattern is characterized by a 
(a)
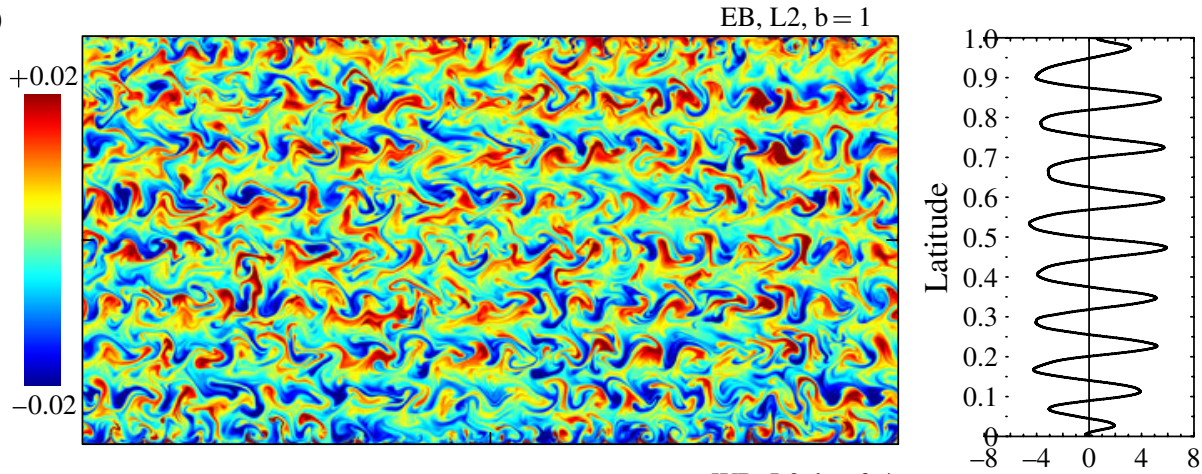

(b)
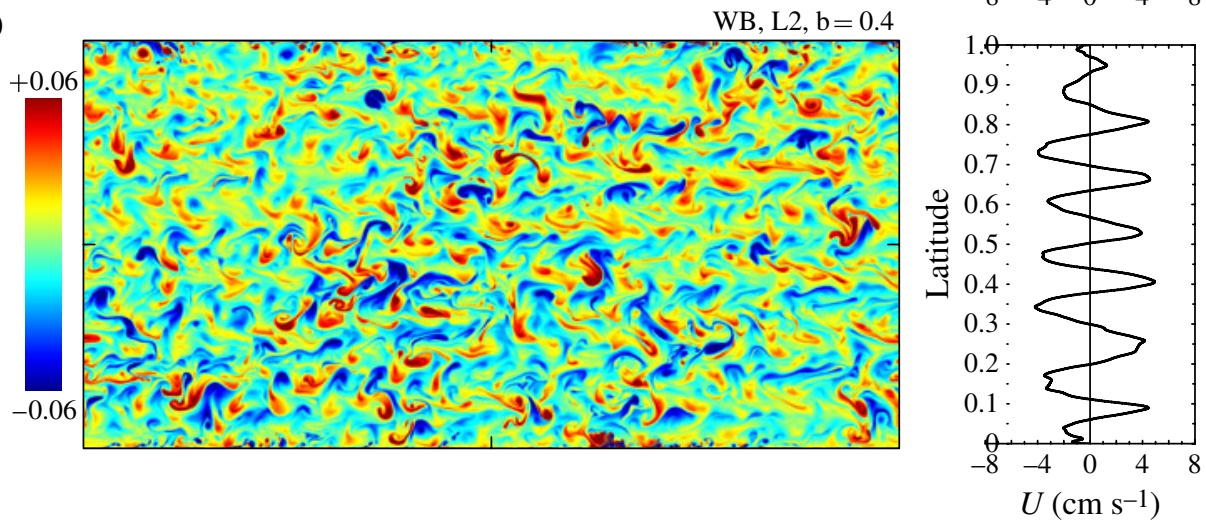

FIgURE 3. Flow in the deep ocean. Deep-ocean PV anomalies of $(a)$ EB and $(b)$ WB flows at intermediate values of the bottom friction, $10^{-7}$ and $4 \times 10^{-8} \mathrm{~s}^{-1}$, respectively. The flow snapshots correspond to figures $1(b)$ and $2(b)$, respectively, but for the deep ocean. Potential vorticity anomaly values are normalized by the Coriolis parameter, and zonally averaged zonal velocity profiles are shown in the right panels.

weakly anisotropic field of coherent eddies. In the deep ocean, the EB jets are always more latent (figure 3).

The WB small-, medium, and large- $\gamma$ cases correspond to $\gamma=10^{-8}, 4 \times$ $10^{-8}, 10^{-7} \mathrm{~s}^{-1}$, respectively, and this range of values is smaller than in the EB case, because the WB jets become essentially non-existent for larger $\gamma$. For the larger values of bottom friction, $\gamma$, believed appropriate for the ocean, we see a significant degree of latency, similar to what is observed in the ocean, whereas for small $\gamma$ we see small degree of latency and very pronounced jets, similar to what is observed in the Jovian atmosphere. The deep-ocean jets in the WB flow are as latent as the upper-ocean ones (figure 6).

An interesting new feature of the large-Re WB solutions is the emergence of very coherent and long-living vortices, which co-exist with less coherent and more transient eddies. Initial PV anomalies in these vortices are generated by viscous stresses on the side boundaries, and as the vortices move away from the boundaries, they mix with ambient fluid and grow in size. (When we replaced the sidewalls with the meridionally periodic boundary condition, the population of the vortices decreased but their intensity increased, and the corresponding PV anomalies got advected across several domain periods rather than getting generated on the sidewalls.) These emerging coherent vortices drift not only to the west, as common for the $\beta$-plane vortices, 
(a)

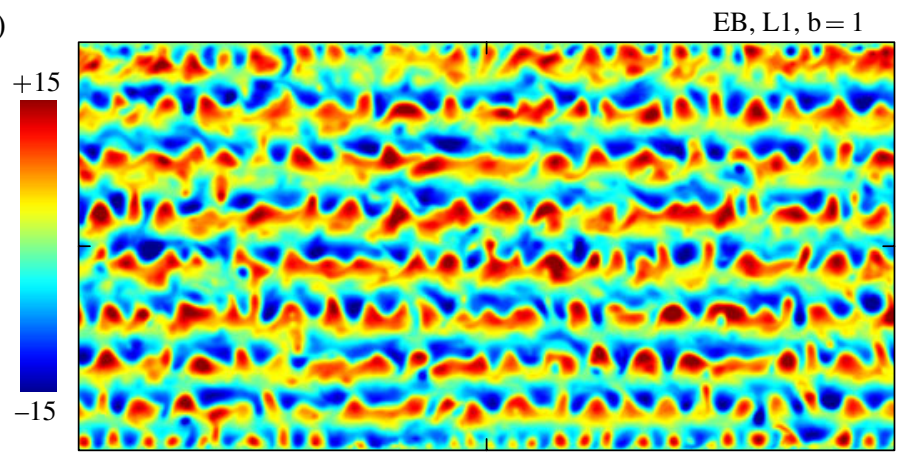

(b)

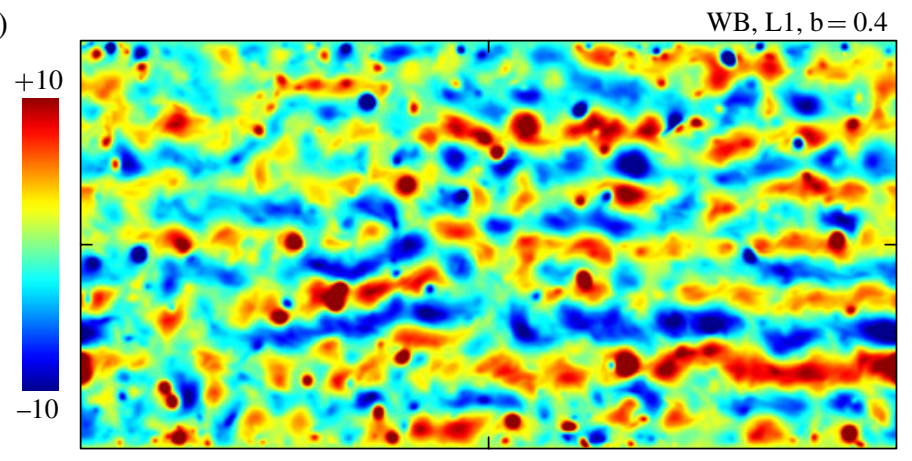

FIGURE 4. Velocity streamfunction of the upper ocean. Inversion of the PV smooths out its fine structure, and the multiple jets become more visible. The flow snapshots correspond to $(a)$ figure $1(b)$ and $(b)$ figure $2(b)$. The streamfunction has been multiplied by the upper layer depth, so units for the colour scale are Sverdrups $\left(10^{6} \mathrm{~m}^{3} \mathrm{~s}^{-1}\right)$.

but also meridionally, and the direction of their meridional drift is always down the background PV gradient, both in the upper and deep layers. Note that McWilliams \& Flierl (1979) reported vortices propagating up the background PV gradient, in seeming contradiction to our results. This difference may be explained partly by structural differences in the vortices - they are dynamically consistent in our case but have an idealized structure in McWilliams \& Flierl (1979). The other important difference is that we focus on a background PV gradient that changes sign in the vertical, whereas McWilliams \& Flierl (1979) considered a vertically uniform one.

An important property of the WB coherent long-living vortices is that they are substantially compensated at depth, that is, their barotropic and baroclinic components compensate each other in the deep layer, and thus the deep-ocean expression of these vortices is weak. Because of this deep compensation, these vortices are relatively insensitive to the bottom friction. However, they are sensitive to the presence of multiple jets (figure 2), because the vortices are less likely to remain intact in the presence of shear dispersion induced by the jets. We leave detailed study of these remarkable large- $R e \mathrm{WB}$ vortices for the future, as this is beyond the limited goals of the present study.

Solutions at low $\operatorname{Re}\left(v=50 \mathrm{~m}^{2} \mathrm{~s}^{-1}\right)$ differ significantly from the large- $R e$ solutions (figures 7 and 8). Both jets and eddies are much weaker, but the jets are also less latent, because the eddies are damped more strongly than the jets by the scaleselective viscosity. The eddies are also in a more orderly arrangement in both zonal 

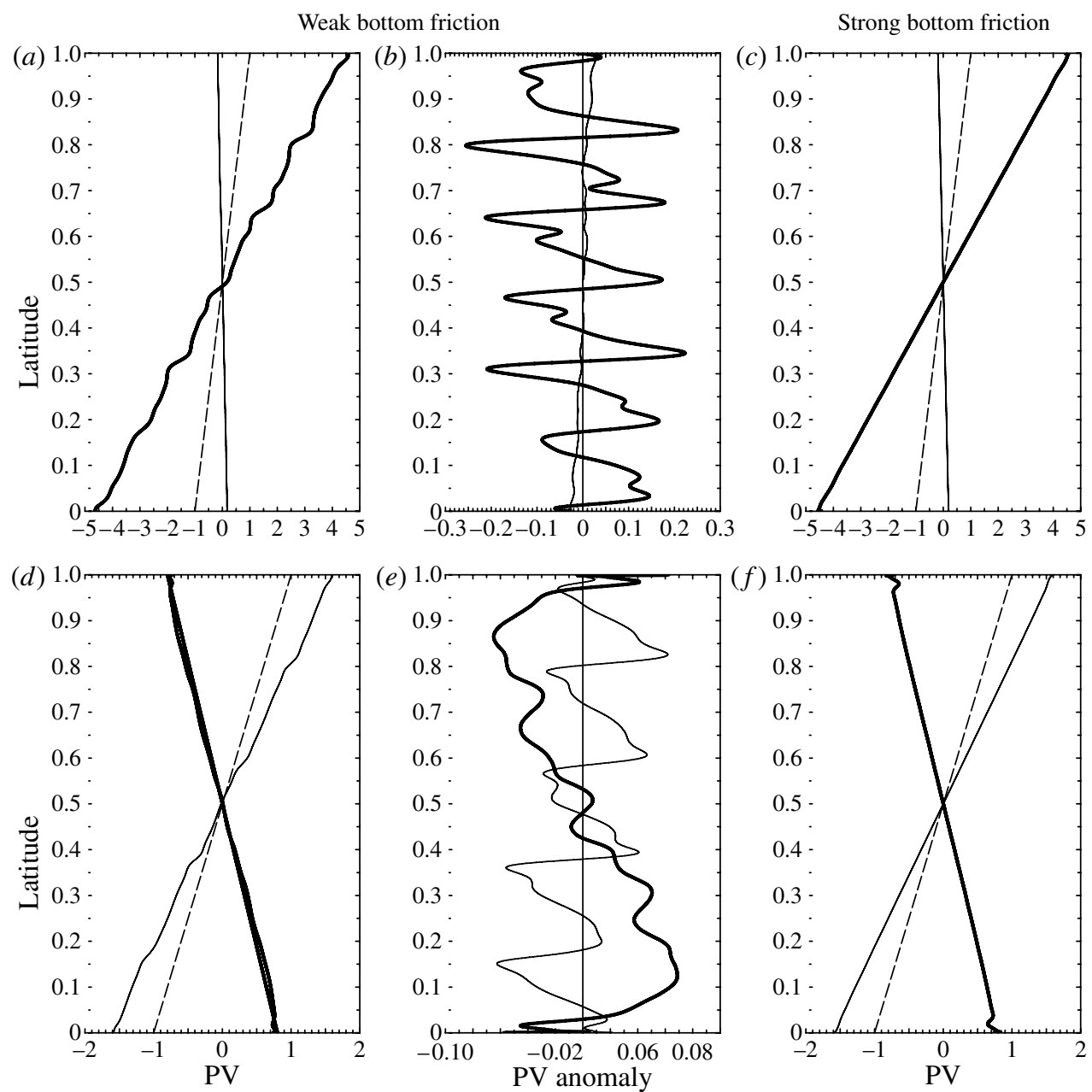

FIGURE 5. Time-mean distributions of PV. Upper-ocean (thick lines) and deep-ocean (thin lines) meridional profiles of the PV and its anomaly are normalized by $\beta$, and the corresponding planetary vorticity, $\beta y$, is indicated by dashed lines. EB $(a-c)$ and WB $(d-f)$ flow solutions at large Reynolds number. Panels $(a, d)$ and $(b, e)$ (PV and PV anomaly profiles, respectively) correspond to the weak bottom friction (figures $1 a$ and $2 a$ ), and panels $(c, f)$ correspond to the large bottom frictions (figures $1 c$ and $2 c$ ).

and meridional directions. Perhaps the biggest difference is that the WB long-living coherent vortices are completely absent.

As figure 6 indicates, and in accord with visual inspection of the corresponding solutions, $\Lambda$ increases with decreasing eddy viscosity, $v$ (i.e. it increases with $R e$ ), and with increasing bottom friction, $\gamma$. While the former tendency is expected, since, as explained above, the scale-selective viscosity affects eddies to a larger degree than the large-scale jets, the latter result is somewhat counterintuitive. The increase of the latency with increasing $\gamma$ is substantial. In the EB case, in both layers there is a reasonably good empirical fit: $\Lambda_{i} \sim \gamma^{0.4}$. In the WB case and at large $R e, \Lambda_{1}$ grows with $\gamma$ more slowly $\left(\sim \gamma^{0.25-0.40}\right)$, but $\Lambda_{2}$ grows faster $\left(\sim \gamma^{0.55-0.65}\right)$. We find that the small-Re WB regime is special, because $\Lambda_{i}$ decays for moderate values of $\gamma$. This 

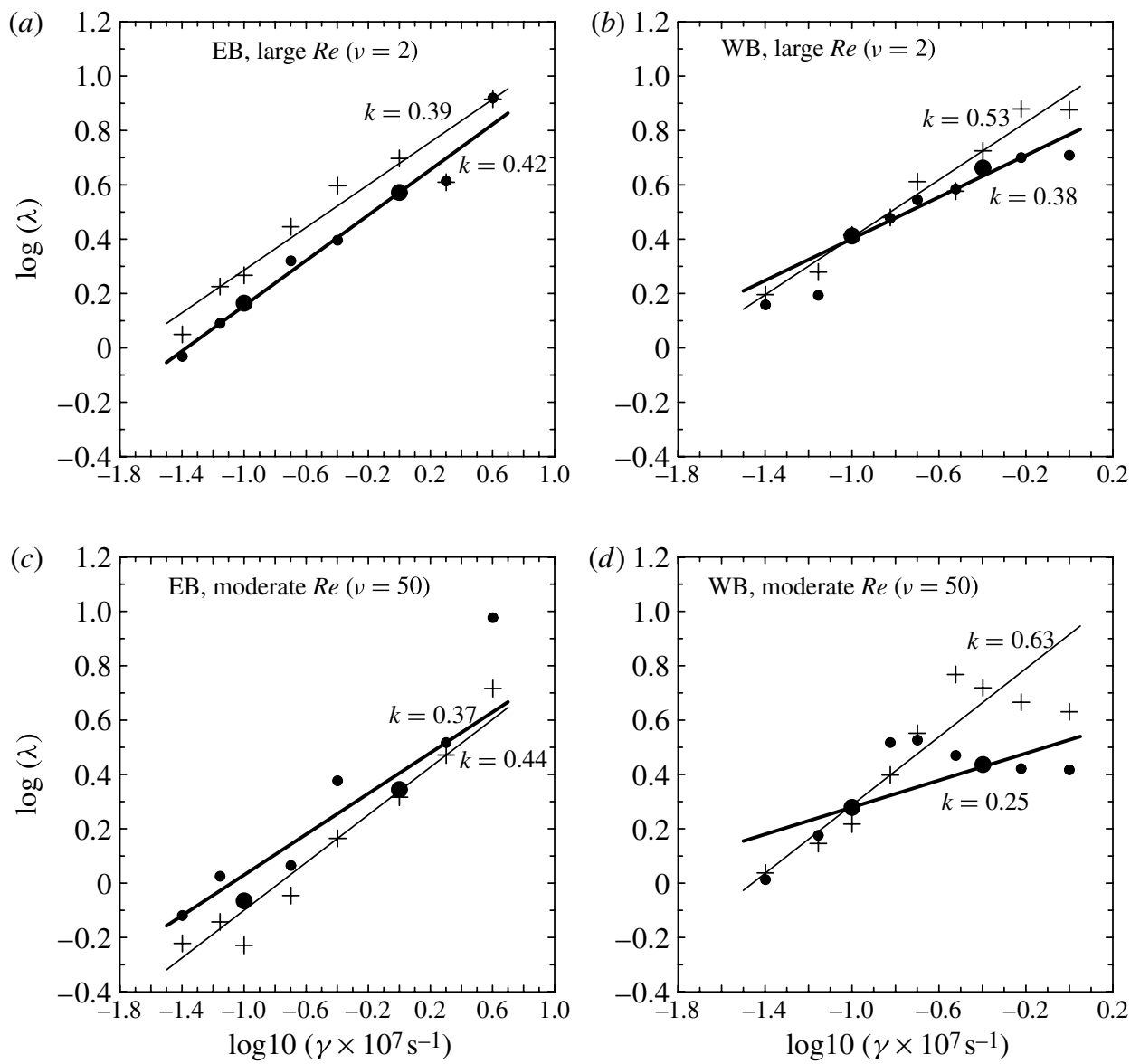

FIGURE 6. Dependence of the latency coefficient, $\Lambda_{i}$, on the bottom friction, $\gamma .(a, b)$ Large-Reynolds-number solutions, $(c, d)$ moderate-Reynolds-number solutions. $(a, c)$ EB flow regimes, $(b, d)$ WB flow regimes. Circles/crosses indicate the values of the latency coefficient in the upper/deep ocean, and the corresponding least-squares fits, $\Lambda_{i} \sim \gamma^{k}$, are shown with the thick and thin lines, respectively. Exponents of the least-squares fits, $k$, are shown next to the corresponding lines. Variations of $\gamma$ are from small, $0.04 \times 10^{-7} \mathrm{~s}^{-1}$, to moderately large values of 4 and $0.8 \times 10^{-7} \mathrm{~s}^{-1}$ in the EB and WB flow regimes, respectively. Values of $\Lambda_{1}$ corresponding to figures $1(b), 2(b), 7(b)$, and $8(b)$ are indicated with larger circles. The breakdown of the trend for the moderate- $R e$ WB case is discussed in $\S 2$.

behaviour is due to the emergence of an upper-layer large-scale flow (not shown) that consists of westward currents near the sidewalls and a broad and weak eastward current in the interior of the channel. The latter current noticeably reduces the unstable vertical shear and thus weakens the eddies and jets. Since this flow regime completely goes away at large $R e$, it is less interesting. Analysis of $\Sigma$ ('jet' PV variance) and $\Sigma^{\prime}$ ('eddy' PV variance) in (2.1) suggests that $\Lambda$ increases with $\gamma$, because, in general, $\Sigma^{\prime}$ increases and $\Sigma$ decreases (not shown). At large $R e$, both $\Sigma^{\prime}$ and $\Sigma$ are decreased for a given value of bottom friction, $\gamma$, indicating that both the eddies and jets are more efficiently damped by the bottom friction (as confirmed by the flow energetics in $\S 3$ ). In the large- $R e \mathrm{WB}$ case, characterized by long-living coherent vortices, the deep-layer $\Sigma^{\prime}$ does not grow with $\gamma$, whereas the corresponding $\Sigma$ has very steep decay (all 
(a)

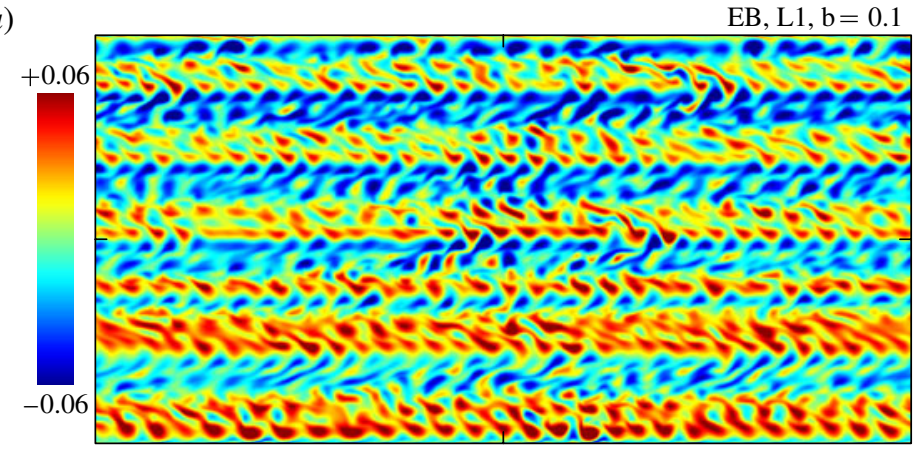

(b)

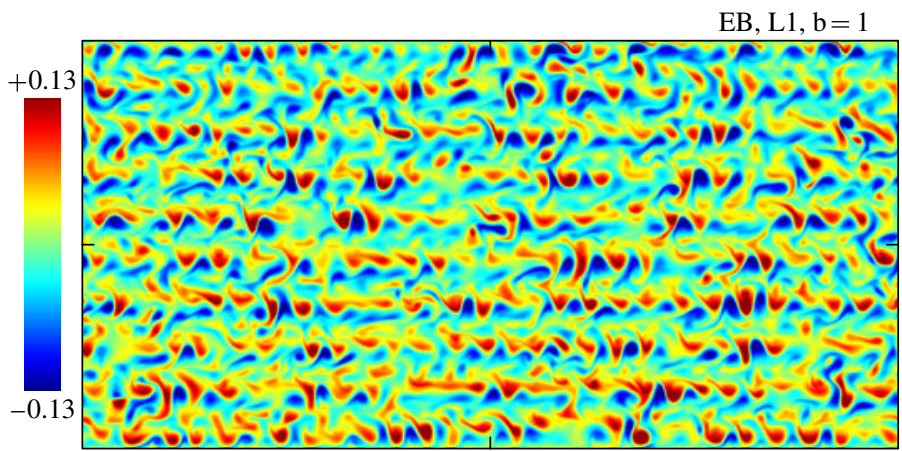

(c)
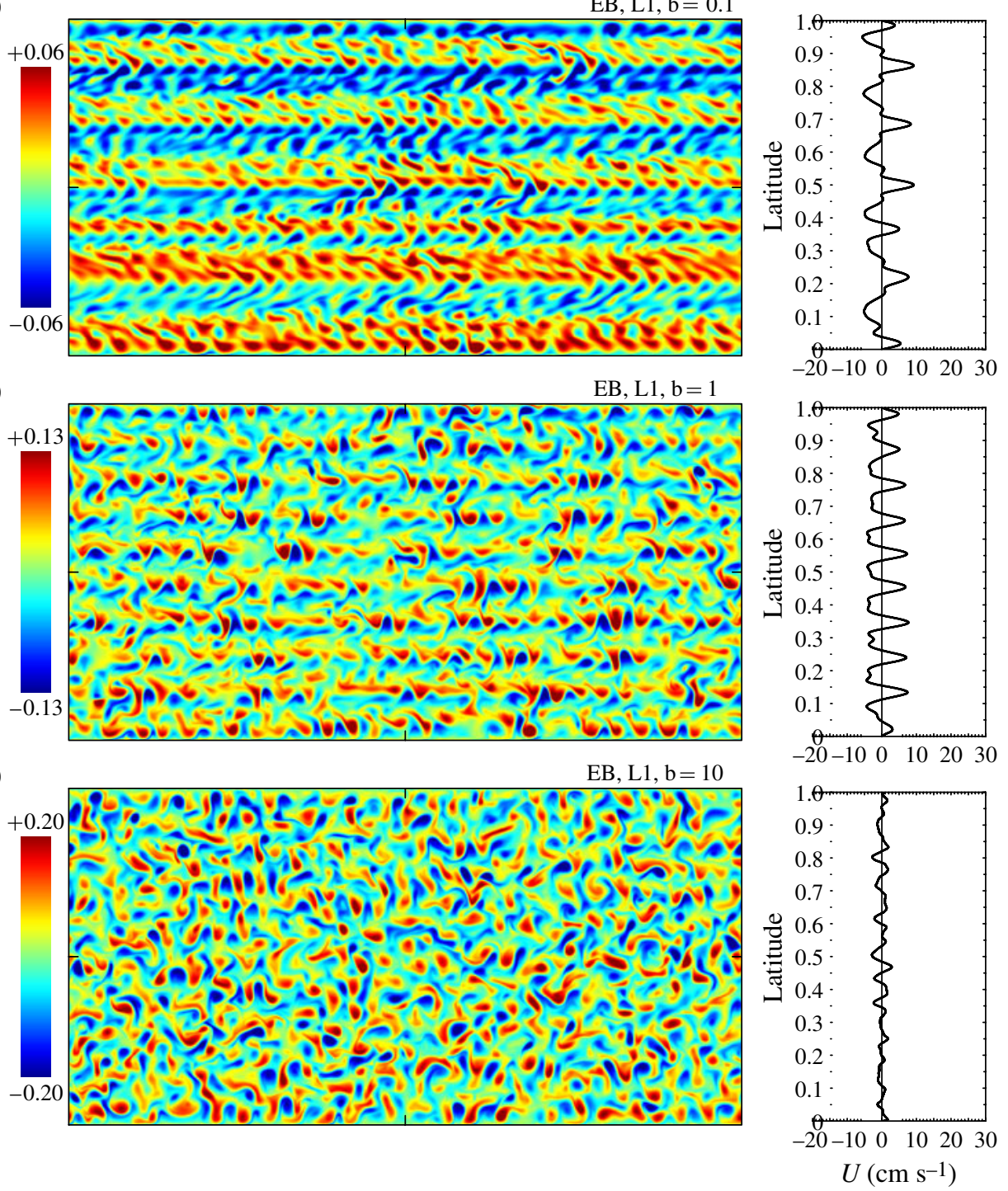

FIgURE 7. Dependence of the multiple jets and eddies on bottom friction: EB flow case, moderate $R e$. Upper-ocean PV anomalies (left panels, in colour) and the corresponding zonally averaged zonal velocity profiles (right panels) are shown for 3 different values of bottom friction, $\gamma$ : (a) $10^{-8},(b) 10^{-7}$, and $(c) 10^{-6} \mathrm{~s}^{-1}$. Potential vorticity anomaly values are normalized by the Coriolis parameter. The parameters for these runs are the same as for the runs shown in figure 1 , except that each run here has $R e=30$ (eddy viscosity is $\left.v=50 \mathrm{~m}^{2} \mathrm{~s}^{-1}\right)$.

consistent with the flow regime that is nearly depth-compensated), and the ratio of the two results in relatively steep growth of $\Lambda_{2}$ (figure $6 b$ ).

Finally, we have checked that our large-Re results do not depend qualitatively on spatial resolution of the grid, by doubling the resolution to $4096 \times 2049$ points. The highest-resolution solutions demonstrated 10\%-20\% increase of $\Lambda$ in all cases, except for the small- $\gamma$ EB case, which showed $10 \%$ decrease of $\Lambda$ associated 
(a)
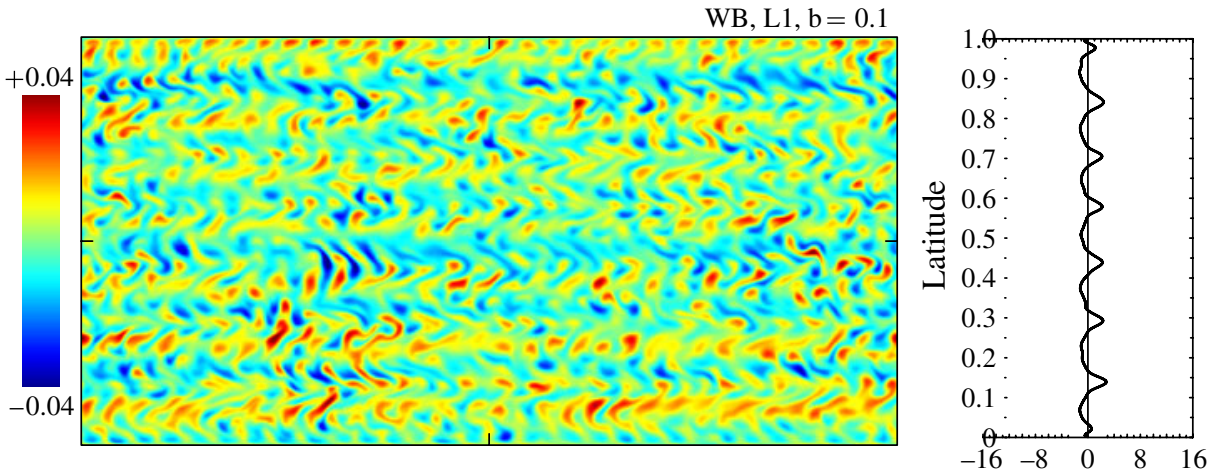

(b)
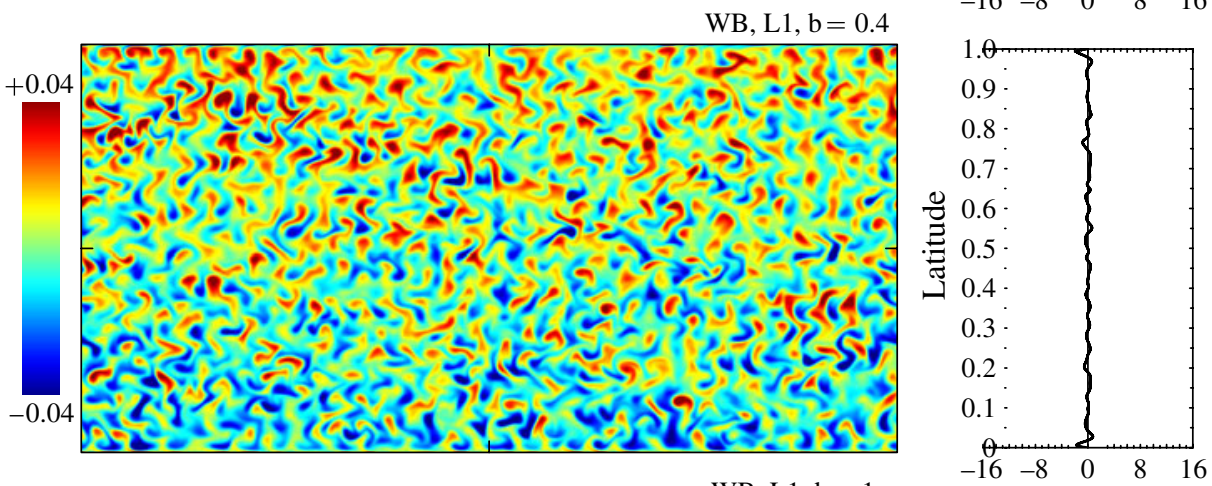

(c)
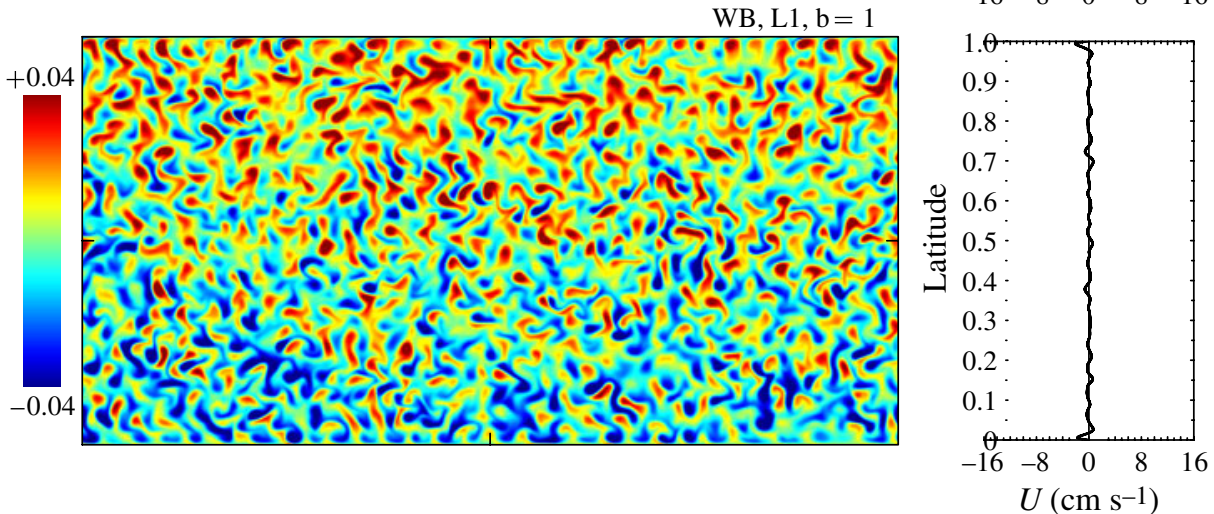

FIGURE 8. Dependence of the multiple jets and eddies on bottom friction: WB flow case, moderate $R e$. Upper-ocean PV anomalies (left panels, in colour) and the corresponding zonally averaged zonal velocity profiles (right panels) are shown for 3 different values of bottom friction, $\gamma:(a) 10^{-8}$, (b) $4 \times 10^{-8}$, and (c) $10^{-7} \mathrm{~s}^{-1}$. Potential vorticity anomaly values are normalized by the Coriolis parameter. The parameters for these runs are the same as for the runs shown in figure 2, except that each run here has $R e=15$ (eddy viscosity is $\left.v=50 \mathrm{~m}^{2} \mathrm{~s}^{-1}\right)$.

with sharpening of the jets. Also, in order to check that the results do not depend qualitatively on the presence of the sidewalls, we looked at solutions of dynamically similar, spatially double-periodic model of the jets and found broadly similar behaviours; the main differences are related to the absence of PV generation on the lateral walls and to more limited meridional migrations of the coherent vortices in 


$\begin{array}{lcccc}\gamma\left(\mathrm{s}^{-1}\right) & 10^{-8} & 4 \times 10^{-8} & 10^{-7} & 10^{-6} \\ \mathrm{~EB}, v=2 & {[1.1(0.2),} & & {[2.0(0.1),} & {[2.0(0.0),} \\ & 3.9(3.0)] & & 20.2(4.2)] & 40.5(0.1)] \\ \mathrm{EB}, v=50 & {[2.0(0.6),} & & {[9.1(0.9),} & {[13.9(0.1),} \\ & 0.5(0.4)] & & 5.6(2.1)] & 35.6(0.6)] \\ \mathrm{WB}, v=2 & {[1.3(0.1),} & {[1.2(0.0),} & {[1.2(0.0),} & \\ \mathrm{WB}, v=50 & 6.4(3.2)] & 13.5(1.1)] & 17.8(0.1)] & \\ & {[1.0(0.2),} & {[2.2(0.0),} & {[2.2(0.0),} & \end{array}$

TABLE 1. Energy dissipation in the nonlinear reference solutions. Each pair of numbers corresponds to the densities of energy dissipation by eddy viscosity and bottom friction, $\left[\overline{D_{v}}, \overline{D_{\gamma}}\right]$, respectively, in units of $10^{3} \mathrm{~m}^{2} \mathrm{~s}^{-3}$. All numbers are rounded to the first decimal digit, and numbers in parentheses show contributions to $\overline{D_{v}}$ and $\overline{D_{\gamma}}$ from the time-mean flow. The numbers in this table correspond to solutions shown in figures $1,2,7$, and 8.

the large- $R e \mathrm{WB}$ case. Thus, the dependences of the latency on the bottom friction and $R e$ are robust.

\section{Analysis of energetics}

In this section we analyse energetics of the flow to scrutinize conclusions obtained in $\S 2$. The energy equation is obtained by multiplying the governing (1.2)-(1.7) by $-\left(H_{i} / H\right) \psi_{i}$ and then summing them to obtain the prognostic equation for the energy density. The viscous and bottom friction terms, then, are rearranged by substituting the identities,

$$
\left.\begin{array}{l}
-\psi \nabla^{2} \nabla^{2} \psi=\nabla \cdot\left(\nabla \psi \nabla^{2} \psi-\psi \nabla \nabla^{2} \psi\right)-\nabla^{2} \psi \nabla^{2} \psi, \\
\psi \nabla^{2} \psi=\nabla \cdot(\psi \nabla \psi)-\nabla \psi \cdot \nabla \psi,
\end{array}\right\}
$$

into the corresponding flux divergence and sign-definite dissipative terms. The basinaveraged dissipation due to the eddy viscosity is

$$
D_{v}=\frac{v}{A} \iint_{A}\left[\frac{H_{1}}{H}\left(\nabla^{2} \psi_{1}\right)^{2}+\frac{H_{2}}{H}\left(\nabla^{2} \psi_{2}\right)^{2}\right] \mathrm{d} A
$$

and the basin-averaged dissipation due to the bottom friction is

$$
D_{\gamma}=\frac{\gamma}{A} \iint_{A} \frac{H_{2}}{H}(\nabla \psi)^{2} \mathrm{~d} A,
$$

where $A$ is the area of the domain. Furthermore, with the velocity streamfunction decomposed into the time-mean and (eddy) fluctuation components, the time-mean $\overline{D_{v}}$ and $\overline{D_{\gamma}}$ have separate contributions due to the time-mean flow and eddies. We calculate these quantities for all reference solutions (table 1) and use them to characterize the flows. The time-mean total dissipation is always equal to the time-mean energy forcing, which is supplied by the unstable background flow. This forcing differs for different solutions, but the general tendency is simple: first, smaller $v$ and smaller $\gamma$ are associated with weaker energy forcing and dissipation; and, second, with larger $\gamma$, the total dissipation is dominated by the eddies (rather than the time-mean jets) and by the bottom (rather than lateral) friction. 
What is the effect of large Re on the energy dissipation? When we increase $R e$ by the factor of 25, the nature of the dissipation changes fundamentally: domination of the eddy viscosity is replaced by domination of the bottom friction, even at moderate values of the bottom friction coefficient (table 1). One could, for example, imagine a completely different dynamical response, in which the flow increased its velocity gradients (e.g. by sharpening the instantaneous jets, as in the 'PV staircase' scenario in Dritschel \& McIntyre 2008) and continued to dissipate laterally, but apparently this scenario is dynamically inconsistent for even moderate values of the bottom friction, $\gamma$. However, for very small values of $\gamma$, that are arguably not oceanic, our large-Re solutions show tendency towards 'PV staircase' in the EB regime (figure 1a), as indicated by very sharp and meandering PV fronts corresponding to the eastward jet cores. The increase of $R e$ also leads to the increase of dissipation (and forcing): by a factor of 4-5 in the WB case (due to emergence of strong coherent vortices) and by a factor of 2 in the small- $\gamma$ EB case (due to emergence of strong jets). Although the eddy viscosity dissipation is linearly proportional to $v$, the change of $v$ from 50 to $2 \mathrm{~m}^{2} \mathrm{~s}^{-1}$ does not lead to 25 -fold decrease of the dissipation. Instead, the viscous dissipation is generally reduced by a smaller factor of $2-7$, and in the small- $\gamma$ WB case it is even increased. This implies that in these cases the relative vorticity of the eddies (in (3.2)) increases by a factor of $2-4$. Thus, generation of the eddy vorticity does not fully compensate for the reduction of the eddy viscosity, and the main mechanism of the energy dissipation switches to the bottom friction. We also note that at large $R e$ the rate of increase of the bottom dissipation with $\gamma$ is slower than at small $R e$, because of the deep compensation.

Energetics of the reference solutions are shown in table 2, and their analysis prompts the following conclusions, which are consistent with analyses of $\S 2$. As expected, we find that larger $R e$ results in more energetic flows, but more so in the WB case, because of the contribution from the intense and long-living coherent vortices. What is unexpected is that the increased bottom friction removes energy preferentially from the mean flow, that is, from the zonal jets. Moreover, in the EB case, the eddies become substantially more energetic with increasing bottom friction, $\gamma$, and this is associated with increasing upper-ocean kinetic and potential energies, and with decreasing deep-ocean kinetic energy. Although this effect may seem similar to the equivalent-barotropization effect in response to large bottom friction in the absence of the $\beta$-effect (Arbic \& Flierl 2004), the solutions are not only far from being equivalent-barotropic but also bottom dissipation dominates because of the deep eddies. In the low-Re EB case this effect is similar but weaker. In the WB solutions we also find a partial equivalent-barotropization effect in the coherent vortices, but since in this flow regime a large fraction of the energy is in the form of the deepocean kinetic energy not associated with these vortices, the total energy decreases with larger $\gamma$.

\section{Linear stability analysis}

In this section, we provide an explanation of the main results obtained from the analysis of nonlinear flow solutions (§2). Following the approach of Berloff et al. $(2009 b)$, we study linear stability properties of the time-mean jets, and the novelty of the analysis here is that we aim to explain the observed dependences of the jet latency on the bottom friction, $\gamma$, and Reynolds number, $R e$. Although all flow regimes seem to be highly nonlinear, our analysis supports the main claim of Berloff et al. (2009b), that these flows are still largely controlled by the footprints of the underlying linear 


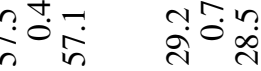

니 용

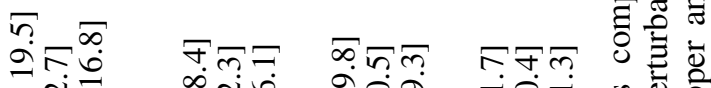

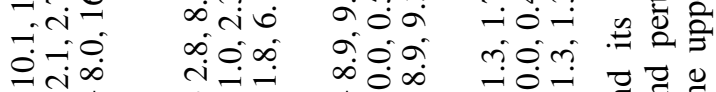

il m+a $\quad+++$ กั่ป்

" $\|$

I|

aัก

$+10-$

ซ్తి

ரோ

ते

|| || $\|$ || ||

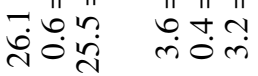

वे 的

¿ ठํㅇㅇ

๙ํำ ๓ำ

$\infty 0+10-$

$\dot{0}+\dot{0} \dot{0}$

$\infty 00$

बे है के

$\stackrel{\infty}{1}$

$++\overrightarrow{+}+\overrightarrow{+}$

nt. non

등

|| || $\mid$

के त्?

|| || ||

लंख़ं

党

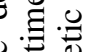

约

$\dot{\tilde{\Xi}}$

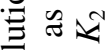

ช 3

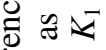

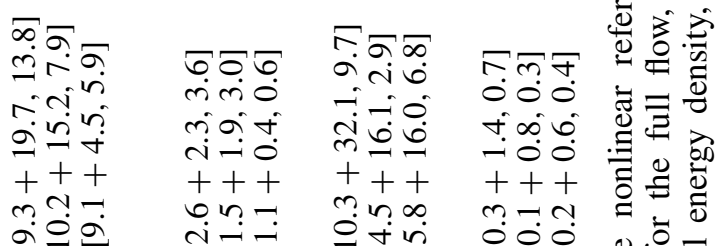

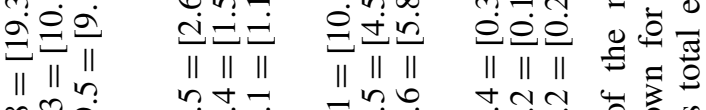

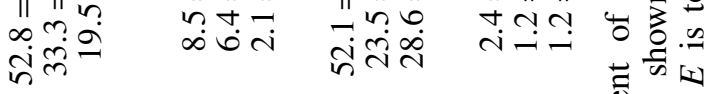

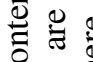

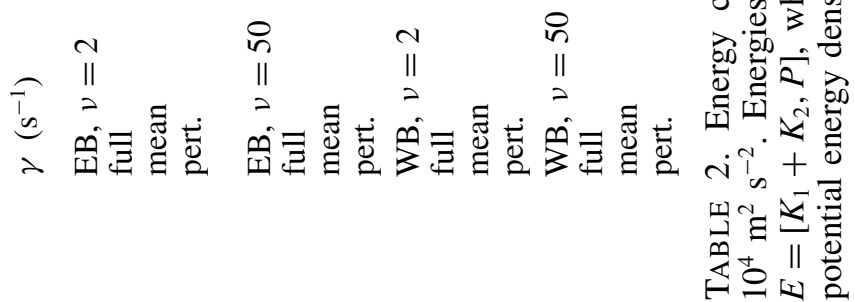



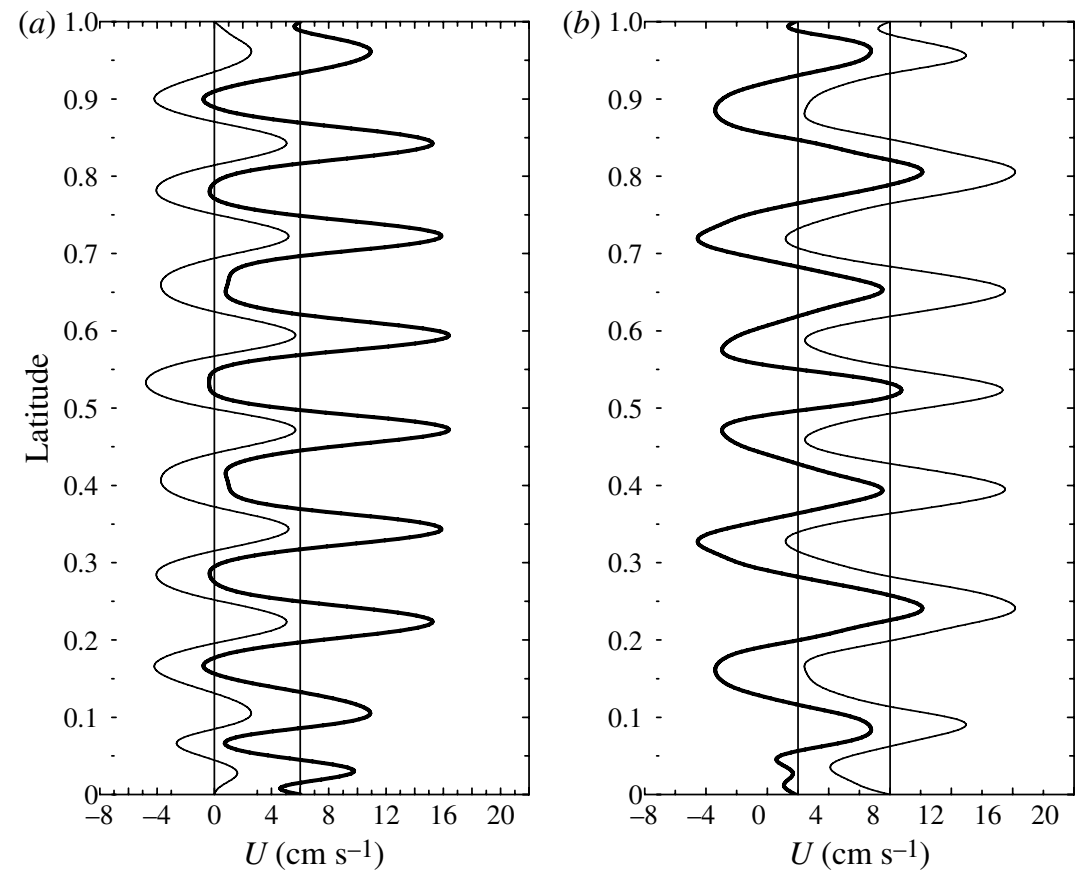

FIGURE 9. Average flow states used for linear stability analysis. Velocity profiles in the upper (thick curve) and deep (thin curve) isopycnal layers corresponding to the (a) EB and (b) WB flow regimes. Straight thin lines indicate velocities of the background flow.

eigenmodes that contribute not only to the eddy patterns but also to the associated nonlinear eddy forcing that maintains the jets. Here, we take this interpretation further by showing that not only these properties but also the latency of the jets is dependent on the underlying linear eigenmodes. In general, large bottom friction could control the latency through the following mechanisms: (a) by weakening the eddy amplitude, (b) by decreasing efficiency of the eddy forcing (i.e. correlation of it with the jets), and (c) by damping zonally uniform eigenmodes (i.e. those that have no structure along the channel) that form the template of the jets. Mechanism (a) cannot explain the increase in latency, because the eddy amplitude actually increases as the eddies become more energetic with larger bottom friction, $\gamma$. The relative contributions of the other two mechanisms are examined below.

The linearized governing equations of the two-layer dynamics are

$$
\begin{gathered}
\frac{\partial q_{1}}{\partial t}+J\left(\Psi_{1}, q_{1}\right)+J\left(\psi_{1}, Q_{1}\right)+\beta \psi_{1 x}=v \nabla^{4} \psi_{1}, \\
\frac{\partial q_{2}}{\partial t}+J\left(\Psi_{2}, q_{2}\right)+J\left(\psi_{2}, Q_{2}\right)+\beta \psi_{2 x}=v \nabla^{4} \psi_{2}-\gamma \nabla^{2} \psi_{2},
\end{gathered}
$$

where we have linearized about $Q_{i}$ and $\Psi_{i}$, the time-mean and zonally averaged PV and velocity streamfunction, respectively, taken from the solutions discussed in $\S 2$. We used two input states corresponding to the EB and WB regimes (figure 9), that are spatially smoothed approximations of the large- $R e$ medium- $\gamma$ solutions (figures $1 b$ and $2 b)$.

We are thus linearizing around a meridionally varying flow that consists of the uniform background flow (first term below) and the finite-amplitude zonal jets 
(second term):

$$
\Psi_{i}=-U_{i} y-\int_{0}^{y} \bar{u}_{i}(y) \mathrm{d} y .
$$

The perturbation streamfunction is Fourier-transformed temporally and zonally,

$$
\psi_{i} \longrightarrow \psi_{i}(y) \exp [\mathrm{i}(k x-\omega t)],
$$

and the Fourier-transformed linearized equations are found:

$$
\begin{aligned}
\omega\left[\psi_{1}^{\prime \prime}-\left(k^{2}+S_{1}\right) \psi_{1}+S_{1} \psi_{2}\right]= & \mathrm{i} \nu \psi_{1}^{I V}+k\left(U_{1}+\bar{u}_{1}-i 2 k v\right) \psi_{1}^{\prime \prime} \\
& +\left[k\left(\beta-k^{2}\left(U_{1}+\bar{u}_{1}\right)-\bar{u}_{1}^{\prime \prime}-S_{1}\left(U_{2}+\bar{u}_{2}\right)\right)\right. \\
& \left.+\mathrm{i} \nu k^{4}\right] \psi_{1}+\left[k S_{1}\left(U_{1}+\bar{u}_{1}\right)\right] \psi_{2}, \\
\omega\left[\psi_{2}^{\prime \prime}-\left(k^{2}+S_{2}\right) \psi_{2}+S_{2} \psi_{1}\right]= & \mathrm{i} \nu \psi_{2}^{I V}+\left[k\left(U_{2}+\bar{u}_{2}-i 2 k v\right)-i \gamma\right] \psi_{2}^{\prime \prime} \\
& +\left[k\left(\beta-k^{2}\left(U_{2}+\bar{u}_{2}\right)-\bar{u}_{2}^{\prime \prime}-S_{2}\left(U_{1}+\bar{u}_{1}\right)\right)\right. \\
& \left.+\mathrm{i}\left(\nu k^{4}+\gamma k^{2}\right)\right] \psi_{2}+\left[k S_{2}\left(U_{2}+\bar{u}_{2}\right)\right] \psi_{1} .
\end{aligned}
$$

The above equations were discretized with second-order finite differences using the same meridional resolution as in the full nonlinear model, the no-slip boundary conditions were applied on the sidewalls, and the resulting generalized eigenproblem was solved numerically. We tested that the outcome is not sensitive to further refinement of the spatial grid. The solutions were obtained in terms of the eigenmode patterns for each zonal wavenumber and the eigenvalues that consist of growth rates and temporal frequencies of the eigenmodes. For each eigenmode we also computed the meridional profile of its temporally and zonally averaged nonlinear self-interaction, which is referred to as the corresponding eddy forcing. Spatial correlation between the eddy forcing and the input state can serve as a measure of the efficiency of the eddy forcing to maintain or destroy the jets.

An important set of linear eigenmodes is the one that corresponds to $k \rightarrow 0$ : these are zonally uniform eigenmodes which have no structure in the zonal direction but oscillate in the meridional direction. It can be easily seen from (4.5)-(4.6) that zonally uniform eigenmodes do not depend on the time-mean zonal velocity, and are thus the same for the EB and WB cases. Some of these eigenmodes are predominantly barotropic, while others are predominantly baroclinic. All of the zonally uniform eigenmodes are damped (i.e. stable), but many of them have very long decay times. We sort these eigenmodes in terms of a decay rate index: the least damped eigenmode has index 1, the second least damped has index 2, and so on. Dependences of the decay time on the mode index for different values of bottom friction, $\gamma$, and eddy viscosity, $\nu$, are shown in figure 10 and discussed further below. Some of the zonally uniform eigenmodes are very efficiently excited by the eddies, and, eventually, they constitute the time-mean jets (Berloff et al. 2009b). We find that larger $\gamma$ shortens the decay times and thus damps out the eigenmodes and makes the jets more latent. Predominantly barotropic zonally uniform eigenmodes are more sensitive to $\gamma$ (figure 10), and the WB multiple jets are dominated by these eigenmodes. This partially explains the observed enhanced sensitivity of the jets of the WB flow to increasing $\gamma(\S 2)$.

We now turn our attention to propagating eigenmodes with zonal structure $(|k|>0)$. Dispersion relationships provide a useful description of the phase propagation and growth rate properties of these eigenmodes (figures 11 and 12). (For convenience, we trade negative eigenfrequency for negative zonal wavenumber, and thus dispersion 

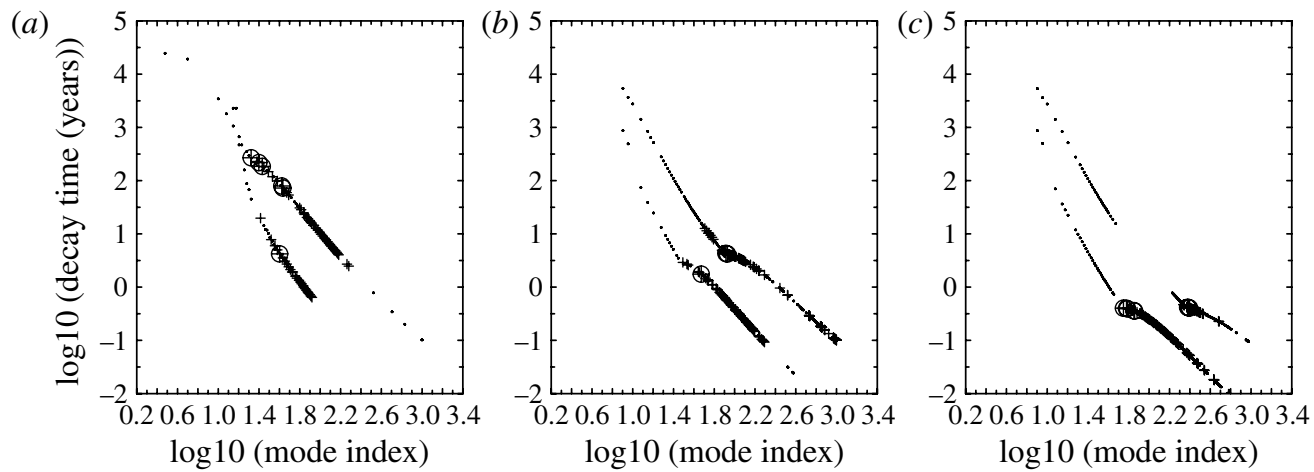

FIGURE 10. Damping of the zonal eigenmodes by bottom friction. The values of the bottom friction are $(a)$ zero, $(b) 10^{-8}$, and $(c) 10^{-7} \mathrm{~s}^{-1}$. The modes are ordered in accordance with their decay rates: a higher mode index corresponds to a shorter decay time. Predominantly baroclinic modes are indicated with dots, and predominantly barotropic modes with crosses. The modes with the eddy forcing that is significantly correlated with the WB flow jets are indicated with large circles with crosses inside (all of these modes are predominantly barotropic): the corresponding decay time substantially shortens with increasing bottom friction.

curves with negative zonal wavenumbers correspond to the patterns propagating to the west.) In most of the theories dealing with the multiple jets, dispersion relationships obtained from linearization around the time-mean state are typically overlooked, perhaps because of the common belief that the flow is too nonlinear. Here, we take advantage of the dispersion relationships, because they help to classify and accurately track the linear eigenmodes, and because they clearly demonstrate the weakening and ultimate demise of some of the eigenmodes in response to increasing bottom friction, $\gamma$. On the $k-\omega$ plots the dispersion relationships are represented by the sets of curves that correspond to the eigenmodes described by the meridional profiles of their amplitude and phase. Along each dispersion curve, the corresponding eigen-pattern changes but nevertheless preserves its main features. The dispersion curves can be sorted into three dynamically distinct types that correspond to the type-1, type-2, and type-3 eigenmodes discussed in Berloff et al. (2009b). Below, we explore dependence of the dispersive properties of the eigenmodes on the dissipative parameters.

Nonlinear extension of the analysis of an eigenmode is based on calculating its eddy forcing (i.e. its nonlinear self-interaction), that can be used for understanding eddy feedback on the multiple jets. In the nonlinear solutions with weak and moderate values of bottom friction, $\gamma$, we find that the eddy forcing patterns are qualitatively similar to those reported in Berloff et al. $(2009 a, b)$; therefore we discuss them briefly and focus our attention on the relations of the eddy forcings to the jet latency. The eddy forcing is (minus) divergence of the eddy PV flux, which consists of fluxes of relative vorticity,

$$
R_{i}=\nabla^{2} \psi_{i}
$$

and isopycnal stretching,

$$
B_{1}=S_{1}\left(\psi_{2}-\psi_{1}\right), \quad B_{2}=S_{2}\left(\psi_{1}-\psi_{2}\right) .
$$



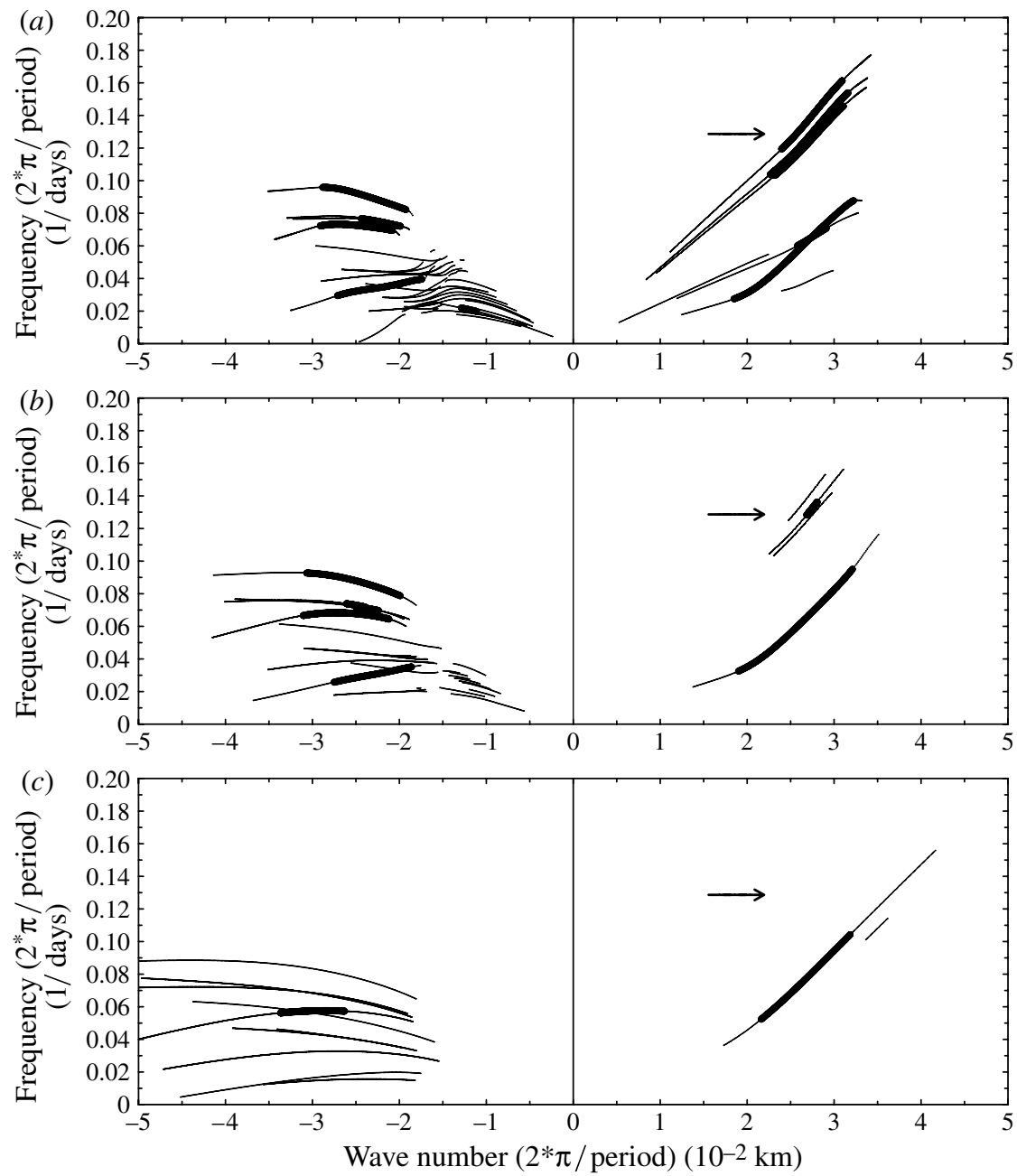

FIGURE 11. Dispersion properties of the linear eigenmodes in the EB flow case. Dependence of zonal-wavenumber/frequency dispersion curves on the bottom friction. The corresponding flow velocity profile is shown in figure $9(a)$. The values of the bottom friction, $\gamma$, are $(a)$ $10^{-8},(b) 10^{-7}$, and $(c) 10^{-6} \mathrm{~s}^{-1}$. Dispersion curves define relationships between real part of the eigenmode frequency (ordinate) and zonal wavenumber (abscissa). Only segments of the dispersion curves that correspond to substantially positive growth rates (i.e. those with inverse imaginary part of the eigenfrequency - growth time scale - that is shorter than 2000 days) are shown; and the most unstable sections (growth time scale shorter than 250 days) are indicated by thicker curves. The arrows indicate the family of the eigenmodes that become stabilized by the bottom friction.

The time-mean (indicated by overbar) and zonally averaged (indicated by angular brackets) eddy forcing,

$$
F_{i}(x, y)=-\left\langle\nabla \overline{\boldsymbol{u}_{i}^{\prime} q_{i}^{\prime}}\right\rangle
$$

can be interpreted as internally generated PV forcing that is balanced by dissipation (Berloff et al. 2009a), and it can be split into divergence of the relative vorticity flux 

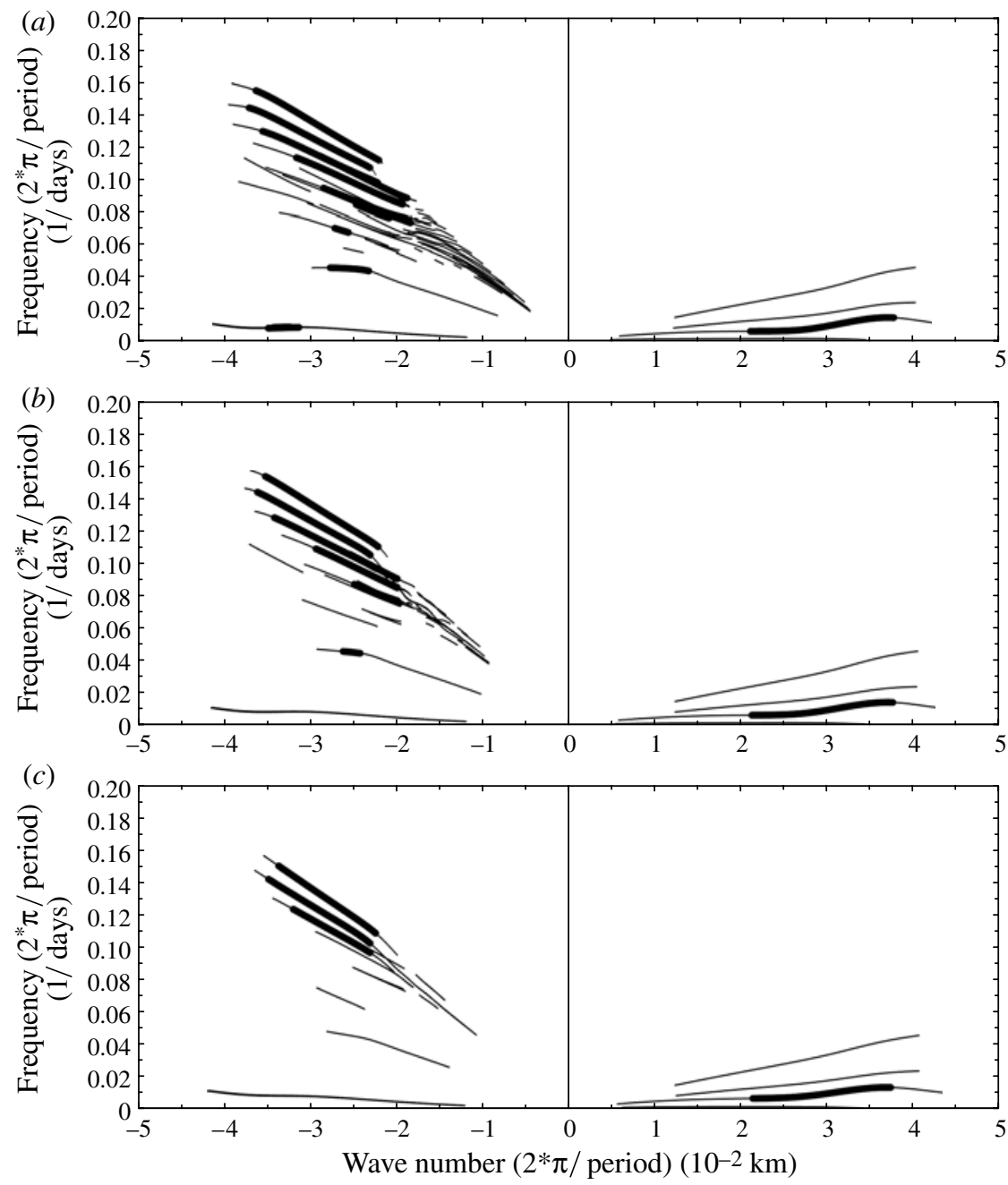

FIGURE 12. Dispersion properties of the linear eigenmodes in the WB flow case. The same as figure 11 , but with the velocity profile shown in figure $9 b$, and with the values of the bottom friction, $\gamma:(a) 10^{-8},(b) 4 \times 10^{-8}$, and $(c) 10^{-7} \mathrm{~s}^{-1}$.

(i.e. Reynolds stress forcing) and divergence of the isopycnal stretching flux (i.e. from stress forcing).

Examples of the three types of most unstable eigenmodes are shown in figure 13 . All these eigenmodes are vertically mixed (i.e. they are barotropic-baroclinic) and upper-ocean-intensified. Many of these eigenmodes are non-local in the meridional direction, in the sense that they straddle several jets. The weaker the jets are, the more non-local are the eigenmodes, and for weak jets all three types merge meridionally into the very non-local 'noodle' mode of instability (Berloff et al. 2009b). The type-1 and type-2 eigenmodes, describing meandering and vascillation, respectively, of the westward jets, propagate to the west and therefore occupy the left halves of the dispersion diagrams (figures 11 and 12). The type-3 eigenmodes occupy the right halves of the dispersion diagrams, and in the WB case there are also type- 3 eigenmodes that slowly propagate to the west (shown by the negative- $k /$ small- $\omega$ branch 
(a)

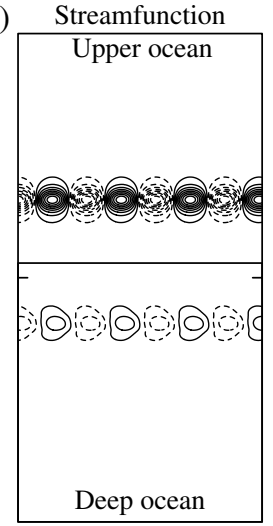

(e)

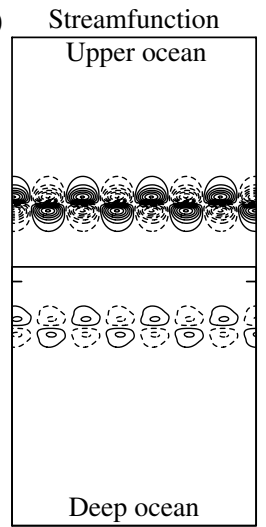

(b)

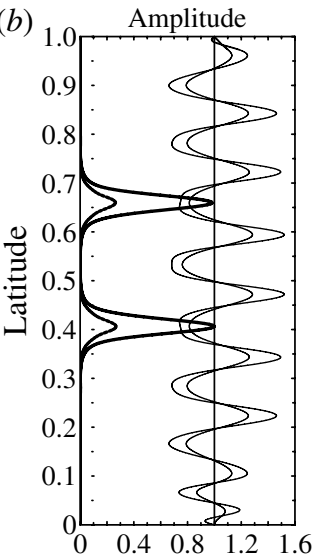

(c) 1.0 BRT Eddy forcing

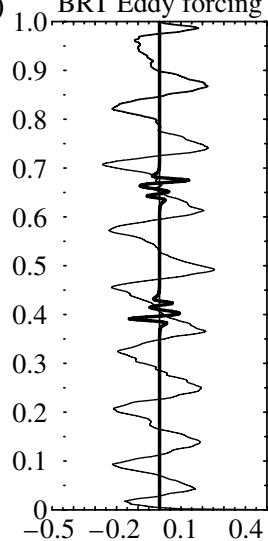

(d) 1.0 BCL Eddy forcing

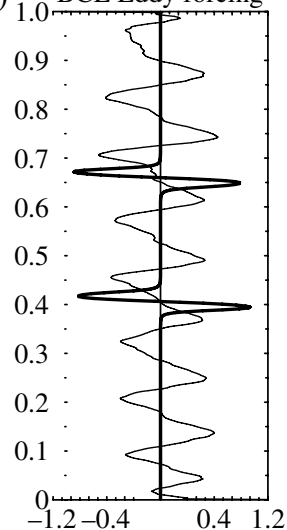

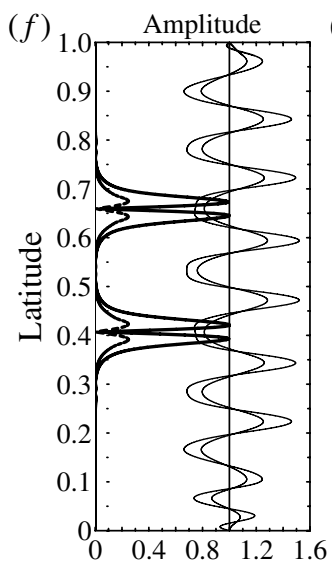

(g) BRT Eddy forcing

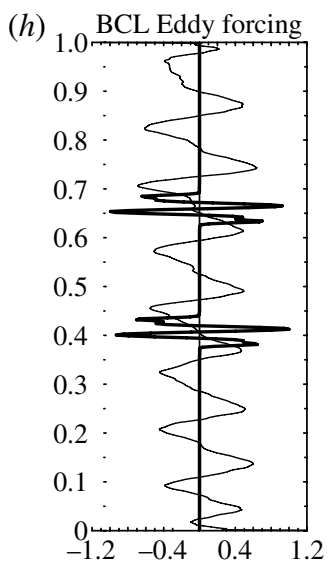

FIGURE 13. For caption see facing page.

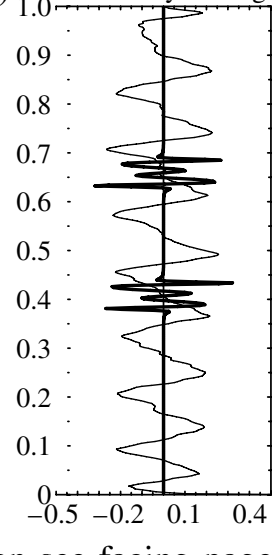

in figure 12). The type-3 eigenmodes correspond to banana-shaped eddies straddling the eastward jets and characterized by strong vertical phase shifts. In the EB and WB cases, the eigenmodes have significant differences in terms of their growth rates and relative importance.

We find that eddy forcings of the eigenmodes (figure 13) are very similar to the eddy forcings diagnosed from the nonlinear solutions, and many eddies are similar to the eigenmode patterns. This result was initially reported by Berloff et al. (2009b), where it was suggested that these 'footprints' of the linear eigenmodes may have been due to the relatively low $R e$ in those simulations. Here, we confirm this result for 50 times larger $R e$, which is encouraging robustness. By correlating the eddy forcings with barotropic and baroclinic components of the jets, we find that the type-1 and type-2 eigenmodes induce eddy forcing that projects mostly on the baroclinic component of the jets and acts mostly in the westward jets. Also, by analysing the relative vorticity and isopycnal stretching components of the eddy forcing, we find that the type-2 eigenmodes support the jets mostly through the form stress (associated with the eddy buoyancy flux convergence), whereas the type-1 eigenmodes support the jets through the Reynolds stress (associated with the eddy momentum flux convergence). Both of these eigenmodes project poorly on the barotropic component of 

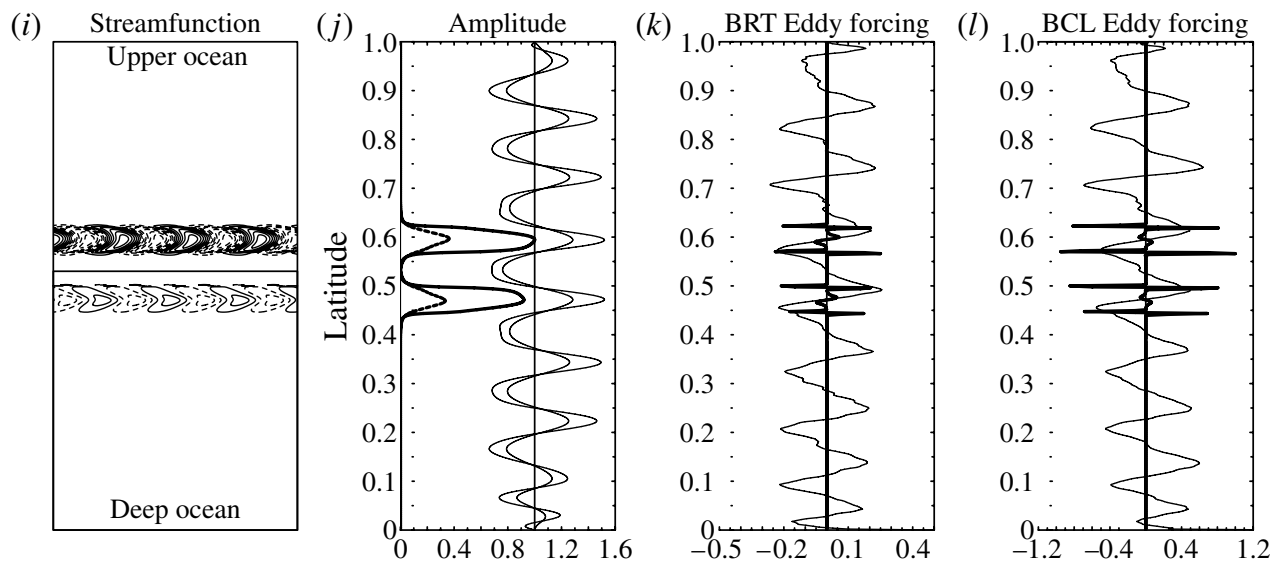

FIGURE 13. Meridionally localized eigenmodes and the corresponding eddy forcing components. Rows $(a-d),(e-h)$ and $(i-l)$ illustrate properties of the type-1, type-2, and type-3 eigenmodes, respectively. Here, the EB flow case is considered, and the eigenmodes belong to the jets positioned in the middle of the channel. Panels $(a),(e)$ and $(i)$ show velocity streamfunctions of the normalized eigenmodes (arbitrary units) in the upper and deep isopycnal layers, illustrated by the upper and lower fractions of the channel (divided by thin line corresponding to the middle of the central westward jet), respectively. Panels $(b)$, $(f)$ and $(j)$ show the amplitudes of the eigenmodes (thick curves; larger/smaller amplitudes correspond to the upper/deep layers), normalized by their maximum upper-ocean value. The corresponding time-mean velocity profiles are scaled by an arbitrary value and shown to the right with thin curves positioned around unity. Panels $(c),(g)$ and $(k)$ show barotropic eddy forcings (thick curves) of the corresponding eigenmodes. Panels $(d),(h)$ and $(l)$ show the corresponding baroclinic eddy forcings (thick curves). Each eddy forcing curve is normalized by the maximum absolute value of the baroclinic eddy forcing, and the corresponding time-mean PV anomalies are shown with thin curves (arbitrary amplitudes).

the jets. Instead, the barotropic component of the jets is supported only by the type- 3 eigenmodes, through their Reynolds stresses. The baroclinic components of the jets are always resisted by the type- 3 eddy forcing but substantially more so in the WB case. The barotropic jets are weakly resisted by the type- 1 eddy forcing in the WB case and by the type- 1 and type- 2 eddy forcings in the EB case.

In addition to damping the zonally uniform eigenmodes that form a template for the jets, large bottom friction can either preferentially damp some unstable eigenmodes, thus reducing their contribution to the full eddy forcing, or modify their meridional structure (i.e. both amplitude and phase) to reduce their eddy forcing efficiencies. With the help of the linear eigenmode analysis, we will now examine the relative importance of these mechanisms in the EB and WB flow regimes. We define the eddy forcing efficiency, $E=\left[E_{B R T}, E_{B C L}\right]$, as the weighted sum of the individual eddy forcing efficiencies associated with all unstable eigenmodes:

$$
\left[E_{B R T}, E_{B C L}\right]=\frac{1}{M} \sum_{n=1}^{n=M} \frac{g_{n}}{g_{\max }}\left[C_{B R T}, C_{B C L}\right],
$$

where the summation is over $M$ eigenmodes with positive growth rates; $g_{n}$ is the growth rate; $g_{\max }$ is the maximum growth rate over the whole set; and $C_{B R T} / C_{B C L}$ are correlation coefficients between the barotropic/baroclinic eddy forcings (defined as the eddy forcing terms in the governing equations projected on the vertical barotropic and 


$$
\begin{array}{lcccc}
\gamma\left(\mathrm{s}^{-1}\right) & 10^{-8} & 4 \times 10^{-8} & 10^{-7} & 10^{-6} \\
\text { EB } & {[0.028,0.023]} & & {[0.015,0.039]} & {[0.001,0.047]} \\
\text { WB } & {[0.005,0.007]} & {[0.007,0.007]} & {[0.010,0.006]} & \\
\text { EB }, U_{B R T}=0 & & & & {[* * *,-0.002]}
\end{array}
$$

TABLE 3. Efficiency of the linear-eigenmode eddy forcing, $\left[E_{B R T}, E_{B C L}\right]$, where $E_{B R T}$ and $E_{B C L}$ are efficiencies of the barotropic and baroclinic eddy forcing components, respectively, for different values of bottom friction, $\gamma$, and for the eddy viscosity value $v=2 \mathrm{~m}^{2} \mathrm{~s}^{-1}$. The last line in the table shows that $E_{B C L}$ is completely degraded when barotropic component of the jets is set to zero. Although $E_{B R T}$ slightly increases with bottom friction in the WB case, the latency still increases, because the zonally uniform eigenmodes become increasingly damped by the bottom friction.

baroclinic modes) and the corresponding PV anomaly profiles. The above definition of the efficiency takes into account the full set of unstable eigenmodes, rather than only the most unstable one, and weights them according to their growth rates. In order to understand the effects of bottom friction and eddy viscosity on the eigenmodes, we linearize about the mean flow of the medium- $\gamma$ solutions (figure 9) for various values of $\gamma$ and $\nu$. The outcome is summarized in table 3 and discussed below.

We will first consider the large- $R e$ solutions with $v=2 \mathrm{~m}^{2} \mathrm{~s}^{-1}$. In the WB case, the linear analysis is relatively simple. The only surprise is that the eddy forcing efficiency is very weak due to the dominance of relatively inefficient type-2 eigenmodes (table 3). As a result, the eddy forcing cannot overcome the damping of the zonally uniform eigenmodes, and the jets become very latent even at moderate values of $\gamma$. This is partly due to the fact that the WB jets are dominated by the barotropic mode, which is more sensitive to the bottom friction. The growth rates of the WB eigenmodes also become smaller with larger $\gamma$, thus contributing to the latency, but we find that there is no preferential damping of any particular type of eigenmode (figure 12). Therefore, the main effect of bottom friction, $\gamma$, on the WB jets is through the increased damping of the zonally uniform eigenmodes rather than altering eddy forcing efficiency. Finally, the long-living coherent vortices that characterize the WB large-Re regime are very different from the linear eigenmodes in terms of their structure and propagation properties, but since these vortices are not correlated with particular jets and propagate nearly intact across many of them, their eddy forcing is not efficient in maintaining the jets.

The large-Re EB case is more complex, because bottom friction induces more complex changes in the eigenmodes and their efficiencies. Unlike in the WB case, the baroclinic component of the EB jets is as strong as their barotropic component, and, therefore, the EB jets are less sensitive to the bottom friction. Also, unlike in the WB case, we find that the EB eddy forcing is rather efficient in maintaining the jets, and the baroclinic efficiency, $E_{B C L}$, even increases with bottom friction, $\gamma$ (table 3). This is due to the dominance of the type-1 and type- 2 eigenmodes that are found to be the least affected by bottom friction. The above facts explain why in the EB case the jets become latent at significantly larger values of $\gamma$. The jets eventually become latent not only because of the damping of the zonally uniform eigenmodes but also because, unlike in the WB case, the bottom friction suppresses all type-3 eigenmodes. The corresponding dispersion relationship branches are indicated 
by arrows in figure 11. The suppression of the type-3 eigenmodes results in dramatic weakening of the barotropic eddy forcing efficiency (table 3) and, hence, in the associated weakening of the barotropic component of the jets. We checked how the weakening of the barotropic component of the jets feeds back on the eigenmodes by putting the barotropic component of the input-state jets to zero. The outcome is the complete loss of the baroclinic eddy forcing efficiency (last line in table 3). Thus, large bottom friction completely erodes eddy feedbacks maintaining the jets.

What happens with the dispersion properties of the eigenmodes at moderate $R e$, that is, for $v=50 \mathrm{~m}^{2} \mathrm{~s}^{-1}$ ? Several changes happen at all considered values of the bottom friction. First, growth rates of the eigenmodes become significantly smaller, and this is consistent with the moderate-Re eddies being weaker and structurally more simple (figures 7 and 8). Second, positions of the dispersion curves on the dispersion diagrams do not change significantly. Third, the type-3 eigenmodes become more damped relative to the other eigenmodes, and this is consistent with noticeable weakening of the barotropic component of the jets in the moderate-Re solutions.

To summarize, in this section we used a combination of the linear arguments, consisting of the analysis of the linear eigenmodes and of its nonlinear extension that considers the nonlinear self-interactions of these eigenmodes and their efficiency in maintaining the jets. We were able to explain qualitatively the observed dependences of the latency on the bottom friction, and the difference between the EB and WB regimes. In the past, sensitivity of a single, eddying baroclinic jet to the bottom friction was studied by Riviere, Treguier \& Klein (2004), and the conclusion was that the linear analysis does not predict flow changes induced by the bottom friction. This discrepancy with our results may be explained, partially, by the differences in the models and parameters. However, a more important reason may be that in our analysis we focused not only on the growth rates of the most unstable modes, but also on the decay rates of the (stable) zonally uniform eigenmodes, and on the efficiency of the nonlinear self-interactions in maintaining the jets.

\section{Conclusions and discussion}

In this study we made a step forward in understanding a remarkable phenomenon in oceanic and atmospheric sciences: co-existence of multiple, nearly zonal jets and the ambient mesoscale eddies. Such jets and eddies are well known from observations of the atmospheres of giant gas planets, such as Jupiter, and much more latent jets are thought to exist in the global ocean $(\S 1.1)$. This study focused on the strength of the multiple jets relative to the strength of the ambient eddies - the 'latency' of the jets - and on the roles of the bottom friction and eddy viscosity in controlling the latency. We find that the degree of the latency is controlled primarily by the bottom friction. The mechanism controlling the latency in our solutions is understood in terms of the changes induced in the linear eigenmodes of the time-mean flow.

The analysis of the effects of dissipation was possible because of the use of a dynamical model that employs a new, highly efficient numerical algorithm. Our model set-up uses parameters appropriate to the ocean, but the dynamical mechanisms discussed may also be active in atmospheric jets.

The nearly zonal, multiple, alternating jets in the oceans are latent, that is, their amplitudes are weak relative to the ambient mesoscale eddies. In contrast, well-known jets on the giant gas planets are very non-latent, that is, they are manifest. Most of the previous studies of the multiple jets focused on their meridional scalings, kinematics and transport properties, shape, and vertical structure, but the importance of 
the latency has been recognized only recently (Kamenkovich et al. 2009a). Factors and mechanisms controlling the latency of the jets have not been systematically studied before, and in this paper we made some progress in this direction. We started by formally defining a latency parameter as the square root of ratio of the jet PV variance to the eddy PV variance. Small latency implies that the jets are easily seen through the ambient eddy field, whereas large latency implies that the jets are masked by more pronounced eddies. Next, we computed a set of solutions for a broad range of the dissipative parameters - lateral eddy viscosity and bottom friction - and analysed it. Bottom friction is found to be the key parameter controlling the latency for all values of the eddy viscosity, despite the fact that flow properties change significantly as a result of the decrease in the eddy viscosity.

The eddy viscosity is scale-selective, and thus the eddies are generally more sensitive to its value than the large-scale jets are; it thus seems reasonable to expect that the latency increases with decreasing eddy viscosity (increasing $R e$ ). On the other hand, an alternative large-Re scenario is that the jets will become sharper and faster, thus reducing the latency; however, we find that this scenario does not occur in the model solutions. The bottom friction is not scale-selective, and at all scales it just damps the most energetic motions in the deep layer; hence it is not obvious a priori that it should act differently on the time-mean jets and the eddies and affect latency. Sorting out these scenarios, as well as predicting magnitudes of the responses to the friction parameters is the focus of this paper. We also considered two different background flow configurations, eastward and westward (denoted EB and WB, respectively), and found not only similarities but also profound differences between these flows and their latency properties. This not only gives us some confidence that we have identified a general effect of bottom friction and indicates what to expect in more realistic flows, but also provides a reference point for more systematic studies of the background flow effects.

Our strategy was to quantify the latency property with the formal latency parameter, $\Lambda$, to vary parameters of the problem, to see which of them have the largest effect on $\Lambda$, and to understand the underlying mechanisms. Our analysis suggests that the degree of the latency is controlled primarily by the bottom friction: the larger the friction, the more latent are the jets; and the degree of latency is significant for the range of the bottom friction coefficient relevant to the real oceans. This result not only provides a plausible explanation for the high degree of latency of the oceanic jets, but also suggests that well-known atmospheric multiple jets on the giant gas planets may be very non-latent because of the absence of a well-defined bottom and the associated bottom friction. Given this result, more attention should be paid to the bottom friction in other models of the multiple jets, but other physical processes (e.g. bottom topography, influence of the lateral boundaries, non-quasigeostrophic effects, etc.) may eventually turn out to be equally or more important. The second important factor was large Reynolds number, Re (i.e. small eddy viscosity), which not only enhances sensitivity of the latency to the increasing bottom friction, but also increases the latency itself.

We focused on a fairly simple, two-layer, quasigeostrophic (QG) model configured on the $\beta$-plane, in a flat-bottomed zonal channel, and driven by an imposed, uniform zonal background flow with vertical shear. This is a classical model (e.g. Haidvogel \& Held (1980); Panetta 1993; Berloff et al. 2009a and 2009b) that simulates multiple, alternating zonal jets, which are believed to be dynamically similar not only to the oceanic but also to the atmospheric jets. In terms of the achieved large $R e$, and thus in terms of the dynamical realism of the eddies and their effects, the flow regimes 
explored here are new. The newly analysed large-Re regime is dynamically more realistic, as its eddy viscosity is in the range of values corresponding to the openocean unbalanced flows (Muller 1976) rather than unresolved parts of the mesoscales. The set of the solutions that we computed allows us to explore the latency for a wide range of $R e$ (i.e. eddy viscosities).

We analysed both EB and WB flows that represent different parts of the midlatitude gyres, and the former also represents the Antarctic Circumpolar Current. Although the emerging patterns of the jets are somewhat similar, the dynamical mechanisms supporting the jets and driven by the eddies differ from those at low values of the bottom friction (Berloff et al. 2009a). In the present study we find that these mechanisms remain fundamentally the same at moderate values of the bottom friction, but cease to exist for strong bottom friction. In terms of the latency, the asymmetry between the EB and WB solutions is very strong, with the EB jets being much less sensitive to the magnitude of the bottom friction. In the large-Re regime, in both EB and WB flows, and at all depths, we find an approximate but robust empirical scaling law for the latency as a function of bottom friction: $\Lambda \sim \gamma^{0.4}$.

The nonlinear results - sensitivity of the latency to the bottom friction and its asymmetry between the EB and WB regimes - are explained via combination of linear analysis and its nonlinear extension, as in the Berloff et al. (2009b) study that focused on formation and meridional scaling of the jets rather than their latency. The linearity enters the problem in terms of the 'footprints' of the most unstable linear eigenmodes that can be clearly seen on the jets. These eigenmodes can nonlinearly self-interact and feed back on the jets that support them. Some of these eigenmodes, having a relatively short zonal scale set by baroclinic instability, act as the drivers supporting the jets. Other eigenmodes that are zonally uniform and weakly damped are excited by the drivers and form the template of the multiple jets. Within this general mechanism, bottom friction can have a profound effect on the latency of the jets by weakening the jets while amplifying the ambient eddies. This can happen for the following reasons: $(a)$ eddy amplitude can be weakened, $(b)$ efficiency of the eddy forcing (i.e. its correlation with the jets) can decrease, and (c) the zonally uniform eigenmodes that form the template of the jets can become more damped. In this paper we sort out relative contributions of $(a)-(c)$ to the latency of the jets. We found that (c) acts both in the EB and WB regimes, whereas mechanism (a) cannot explain the results, because the eddy amplitudes, as well as the associated eddy forcing only intensify with increasing bottom friction. We also explained that the jets in the EB regime are more resistant to the bottom friction because of the strong efficiency of the eddy forcing.

Another outcome of our study is connection between the jet latency and the 'staircase' hypothesis (\$1.1), which predicts emergence of a set of very sharp barriers separated by mixing zones in large-Re jets. One of our conclusions is that the 'staircase' state, characterized by very low latency, can be achieved only with very weak bottom friction. Therefore, it is irrelevant for the oceanic jets, and this is consistent with the previous results $(\S 1.1)$; however, it may be relevant for the atmospheric jets.

Although we find that some essential properties of the eddies can be understood in terms of the self-interacting linear waves, scrutinizing the linear-eigenmode (i.e. linear-wave) interpretations, studying possible wave-triad interactions, and examining the roles of full nonlinearity must be continued. Also, future advances from our highly idealized model will have to be toward more realistic background flows with non-zonal orientation, spatial inhomogeneity and temporal variability, toward 
progressively more realistic bottom topography, toward effects of eastern boundaries, and toward dynamical connection between the equatorial, midlatitude, and highlatitude jet dynamics. Finally, in the WB regime with large $R e$, we encountered intense long-lived coherent vortices generated both in the interior and on the lateral walls. These long-lived vortices are substantially depth-compensated, and, therefore, they are only weakly dissipated by the bottom friction. Systematic study of these vortices, their interactions and transport properties will be the subject of a separate study.

Funding was provided: for P.B. by NSF grants OCE 0725796 and OCE 0845150 , for J.T.F. by NSF grant OCE 0845150, for I.K. by NSF grant OCE 0842834 , and for S.K. by the University Research Fellowship from the Royal Society. S.K. also acknowledges support from the Mary Sears Grant from the Woods Hole Oceanographic Institution and the hospitality of the Institution during his visit. The authors are grateful to anonymous reviewers for their helpful comments.

\section{REFERENCES}

ARBIC, B. \& Flierl, G. 2004 Baroclinically unstable geostrophic turbulence in the limits of strong and weak bottom Ekman friction: application to midocean eddies. J. Phys. Oceanogr. 34, 2257-2273.

ARBIC, B. \& SCOTT, R. 2008 On quadratic bottom drag, geostrophic turbulence, and oceanic mesoscale eddies. J. Phys. Oceanogr. 38, 84-103.

Baldwin, M., Rhines, P., Huang, H.-P. \& McIntyre, M. 2007 The jet-stream conundrum. Science 315, 467-468.

Balk, A., Nazarenko, S. \& Zakharov, V. 1990 On the nonlocal turbulence of drift type waves. Phys. Rev. Lett. A 146, 217-221.

Barnier, B., HuA, B. L. \& Le Provost, C. 1991 On the catalytic role of high baroclinic modes in eddy-driven large-scale circulations. J. Phys. Oceanogr. 21, 976-997.

Berloff, P., Kamenkovich, I. \& Pedlosky, J. 2009a A model of multiple zonal jets in the oceans: dynamical and kinematical analysis. J. Phys. Oceanogr. 39, 2711-2734.

Berloff, P., Kamenkovich, I. \& Pedlosky, J. 2009b A mechanism of formation of multiple zonal jets in the oceans. J. Fluid Mech. 628, 395-425.

Beron-Vera, F., Olascoaga, M., Brown, M., Kocak, H. \& Rypina, I. 2010 Invariant-torilike coherent structures in geophysical flows. Chaos 20, 017514.

Buckingham, C., Cornillon, P. \& Obenour, K. 2011 Zonal bands observed in microwave SST front probability. J. Geophys. Res. (submitted).

Chekhlov, A., Orszag, S., Sukoriansky, S., Galperin, B. \& Staroselsky, I. 1996 The effect of small-scale forcing on large-scale structures in two-dimensional flows. Physica D 98, 321-334.

Chelton, D., Schlax, M. \& Samelson, R. 2011 Global observations of nonlinear mesoscale eddies. Prog. Oceanogr. 91, 167-216.

Connaughton, C., Nadiga, B., Nazarenko, S. \& Quinn, B. 2010 Modulational instability of Rossby and drift waves and generation of zonal jets. J. Fluid Mech. 654, 207-231.

DANILOV, S. \& GURARIE, D. 2004 Scaling, spectra and zonal jets in beta-plane turbulence. Phys. Fluids 16, 2592-2603.

DANILOV, S. \& GRYANIK, V. 2004 Barotropic beta-plane turbulence in a regime with strong zonal jets revisited. J. Atmos. Sci. 61, 2283-2295.

DEWAR, W. 1998 Topography and barotropic transport control by bottom friction. J. Mar. Res. 56, 295-328.

Dritschel, D. \& McIntyre, M. 2008 Multiple jets as PV staircases: the Phillips effect and the resilience of eddy-transport barriers. J. Atmos. Sci. 65, 855-874.

Dritschel, D. \& Scott, R. 2011 Jet sharpening by turbulent mixing. Phil. Trans. R. Soc. A 369, 754-770. 
Farrell, B. \& IoAnnou, P. 2007 Structure and spacing of jets in barotropic turbulence. J. Atmos. Sci. 64, 3652-3665.

Farrell, B. \& IoANnou, P. 2008 Formation of jets by baroclinic turbulence. J. Atmos. Sci. 65, 3353-3375.

FARrell, B. \& IOANNOU, P. 2009 Emergence of jets from turbulence in the shallow-water equations on an equatorial beta plane. J. Atmos. Sci. 66, 3197-3207.

Fureby, C. \& Grinstein, F. 2002 Large eddy simulation of high-Reynolds-number free and wall-bounded flows. J. Comput. Phys. 181, 68-97.

Galperin, B., Nakano, H., Huang, H. \& Sukoriansky, S. 2004 The ubiquitous zonal jets in the atmospheres of giant planets and Earth's oceans. Geophys. Res. Lett. 31, L13303.

Galperin, B., Sukoriansky, S. \& Dikovskaya, N. 2010 Geophysical flows with anisotropic turbulence and dispersive waves: flows with a beta-effect. Ocean Dyn. 60, 427-441.

Goloviznin, V., Glotov, V., Danilin, A., Korotkin, I. \& Karabasov, S. 2011 Computational modelling of incompressible flows in multiple dimensions with the CABARET method. In Basic Problems of Multiphase and Turbulent Flows. Nauka (in press).

Haidvogel, D. \& Held, I. 1980 Homogeneous quasi-geostrophic turbulence driven by a uniform temperature gradient. J. Atmos. Sci. 37, 2644-2660.

Herbei, R., McKeague, I. \& Speer, K. 2008 Gyres and jets: inversion of tracer data for ocean circulation structure. J. Phys. Oceanogr. 38, 1180-1202.

Hogg, N. \& Owens, B. 1999 Direct measurement of the deep circulation within the Brazil basin. Deep-Sea Res. 46, 335-353.

Hristova, H., Pedlosky, J. \& Spall, M. 2008 Radiating instability of a meridional boundary current. J. Phys. Oceanogr. 38, 2294-2307.

Huang, H.-P., Kaplan, A., Curchitser, E. \& Maximenko, N. 2007 The degree of anisotropy for mid-ocean currents from satellite observations and an eddy-permitting model simulation. J. Geophys. Res. 112, C09005.

HuAng, H.-P. \& Robinson, W. 1998 Two-dimensional turbulence and persistent zonal jets in a global barotropic model. J. Atmos. Sci. 55, 611-632.

Hughes, C., Thompson, A. \& Wilson, C. 2010 Identification of jets and mixing barriers from sea level and vorticity measurements using simple statistics. Ocean Model. 32, 44-57.

Ivanov, L., Collins, C. \& Margolina, T. 2008 System of quasi-zonal jets off California revealed from satellite altimetry. Geophys. Res. Lett. 36, L03609.

Kamenkovich, I., Berloff, P. \& Pedlosky, J. 2009a Role of eddy forcing in the dynamics of multiple zonal jets in the North Atlantic. J. Phys. Oceanogr. 39, 1361-1379.

Kamenkovich, I., Berloff, P. \& Pedlosky, J. $2009 b$ Anisotropic material transport by eddies and eddy-driven currents in the North Atlantic. J. Phys. Oceanogr. 39, 3162-3175.

Karabasov, S., Berloff, P. \& Goloviznin, V. 2009 CABARET in the ocean gyres. Ocean Model. 30, 155-168.

Karabasov, S. \& Goloviznin, V. 2009 Compact accurately boundary adjusting high-resolution technique for fluid dynamics. J. Comput. Phys. 228, 7426-7451.

KASPI, I. \& FlierL, G. 2007 Formation of jets by baroclinic instability on gas planet atmospheres. J. Atmos. Sci. 64, 3177-3194.

Kondratyev, K. \& Hunt, G. 1982 Weather and Climate on Planets. Pergamon.

LEE, S. 1997 Maintenance of multiple jets in a baroclinic flow. J. Atmos. Sci. 54, 1726-1738.

Levy, M., Klein, P., Treguier, A.-M., Iovino, D., Madec, G., Masson, S. \& Takahashi, K. 2010 Modifications of gyre circulation by sub-mesoscale physics. Ocean Model. 34, 1-15.

LiU, J. \& Schneider, T. 2011 Mechanisms of jet formation on the giant planets. J. Atmos. Sci. 67, 3652-3672.

Manfroi, A. \& Young, W. 2002 Stability of $\beta$-plane Kolmogorov flow. Physica D 162, 208-232.

Manfroi, A. \& Young, W. 1999 Slow evolution of zonal jets on the beta plane. J. Atmos. Sci. 56, 784-800.

MARIE, L. 2010 A study of the phase instability of quasi-geostrophic Rossby waves on the infinite beta-plane to zonal flow perturbations. Nonlinear Process. Geophys. 17, 49-63. 
Marshall, J., Shuckburgh, E., Jones, H. \& Hill, C. 2006 Estimates and implications of surface eddy diffusivity in the Southern Ocean derived from tracer transport. J. Phys. Oceanogr. 36, 1806-1821.

MAXIMENKO, N., BANG, B. \& SASAKI, H. 2005 Observational evidence of alternating zonal jets in the world ocean. Geophys. Res. Lett. 32, L12607.

Maximenko, N., Melnichenko, O., Nitler, P. \& Sasaki, H. 2008 Stationary mesoscale jet-like features in the ocean. Geophys. Res. Lett. 35, L08603.

MCIntyre, M. 1982 How well do we understand the dynamics of stratospheric warmings? J. Met. Soc. Japan 60, 37-65.

MCWiLliams, J. 1977 A note on a consistent quasigeostrophic model in a multiply connected domain. Dyn. Atmos. Oceans 1, 427-441.

McWilliams, J. \& Flierl, G. 1979 On the evolution of isolated, nonlinear vortices. J. Phys. Oceanogr. 9, 1155-1182.

Melnichenko, O., Maximenko, N., Schneider, N. \& SASAKi, H. 2010 Quasi-stationary striations in basin-scale oceanic circulation: vorticity balance from observations and eddy-resolving model. Ocean Dyn. 60, 653-666.

Muller, P. 1976 On the diffusion of momentum and mass by internal gravity waves. J. Fluid Mech. 77, 789-823.

NAKano, H. \& Hasumi, H. 2005 A series of zonal jets embedded in the broad zonal flows in the Pacific obtained in eddy-permitting ocean general circulation models. J. Phys. Oceanogr. 35, 474-488.

Ollitrault, M., Lankhorst, M., Fratantoni, D. \& Richardson, P. 2006 Zonal intermediate currents in the equatorial Atlantic Ocean. Geophys. Res. Lett. 33, L05605.

PANetTA, L. 1993 Zonal jets in wide baroclinically unstable regions: persistence and scale selection. J. Atmos. Sci. 50, 2073-2106.

Pedlosky, J. 1987 Geophysical Fluid Dynamics, 2nd edn. Springer.

QIU, B., ScOTT, R. \& CHEN, S. 2008 Length scales of eddy generation and nonlinear evolution of the seasonally-modulated South Pacific subtropical countercurrent. J. Phys. Oceanogr. 38, $1515-1528$.

RADKO, T. 2011 On the generation of large-scale structures in a homogeneous eddy field. $J$. Fluid Mech. 668, 76-99.

Read, P., Yamazaki, Y., Lewis, S., Williams, P., Wordsworth, R., Miki-Yamazaki, K., Sommeria, J., Didelle, H. \& Fincham, A. 2007 Dynamics of convectively driven banded jets in the laboratory. J. Atmos. Sci. 64, 4031-4052.

RHINeS, P. 1975 Waves and turbulence on a beta-plane. J. Fluid Mech. 69, 417-443.

Rhines, P. 1994 Jets. Chaos 4, 313-339.

Richards, K., Maximenko, N., Bryan, F. \& SASAKi, H. 2006 Zonal jets in the Pacific ocean. Geophys. Res. Lett. 33, L03605.

Riviere, P., Treguier, A. \& Klein, P. 2004 Effects of bottom friction on nonlinear equilibration of an oceanic baroclinic jet. J. Phys. Oceanogr. 34, 416-432.

Schlax, M. \& Chelton, D. 2008 The influence of mesoscale eddies on the detection of quasi-zonal jets in the ocean. Geophys. Res. Lett. 35, L24602.

van Sebille, E., Kamenkovich, I. \& Willis, J. 2011 Quasi-zonal jets in 3D Argo data of the northeast Atlantic. Geophys. Res. Lett. 38, L02606.

Sinha, B. \& Richards, K. 1999 Jet structure and scaling in Southern Ocean models. J. Phys. Oceanogr. 29, 1143-1155.

Smith, K. 2004 A local model for planetary atmospheres forced by small-scale convection. J. Atmos. Sci. 61, 1420-1433.

Sokolov, S. \& Rintoul, S. $2007 a$ Multiple jets of the Antarctic Circumpolar Current south of Australia. J. Phys. Oceanogr. 37, 1394-1412.

Sokolov, S. \& Rintoul, S. $2007 b$ On the relationship between fronts of the Antarctic Circumpolar Current and surface chlorophyll concentrations in the Southern Ocean. $J$. Geophys. Res. 112, C07030.

Sokolov, S. \& Rintoul, S. 2009 Circumpolar structure and distribution of the Antarctic Circumpolar Current fronts. Part 1. Mean circumpolar paths. J. Geophys. Res. 114, C11018. 
Sukoriansky, S., Dikovskaya, N. \& Galperin, B. 2007 On the arrest of inverse energy cascade and the Rhines scale. J. Atmos. Sci. 64, 3312-3327.

SwarzTrauber, P. 1977 The methods of cyclic reduction, Fourier analysis and the FACR algorithm for the discrete solution of Poisson's equation on a rectangle. SIAM Rev. 19, 490-501.

TheIss, J. 2004 Equatorward energy cascade, critical latitude, and the predominance of cyclonic vortices in geostrophic turbulence. J. Phys. Oceanogr. 34, 1663-1678.

Thompson, A. 2010 Jet formation and evolution in baroclinic turbulence with simple topography. J. Phys. Oceanogr. 40, 257-278.

Thompson, A. \& Young, W. 2007 Baroclinic eddy heat fluxes: zonal flows and energy balance. J. Atmos. Sci. 64, 3214-3231.

Treguier, A. \& PAnetTA, L. 1994 Multiple zonal jets in a quasigeostrophic model of the Antarctic Circumpolar Current. J. Phys. Oceanogr. 24, 2263-2277.

VAllis, G. \& MAltrud, M. 1993 Generation of mean flows and jets on a beta plane and over topography. J. Phys. Oceanogr. 23, 1346-1362.

Wallcraft, A., Birol Kara, A. \& Hurlburt, H. 2005 Convergence of Laplacian diffusion versus resolution of an ocean model. Geophys. Res. Lett. 32, L07604.

Williams, G. 1978 Planetary circulations. Part 1. Barotropic representation of Jovian and terrestrial turbulence. J. Atmos. Sci. 35, 1399-1426.

Yoo, C. \& LeE, S. 2010 Persistent multiple jets and PV staircase. J. Atmos. Sci. 67, 2279-2295. 